Swarthmore College

Works

8-1-2014

\title{
Temporal And Spatial Turbulent Spectra Of MHD Plasma And An Observation Of Variance Anisotropy
}

\author{
D. A. Schaffner
}

Michael R. Brown

Swarthmore College, doc@swarthmore.edu

Vyacheslav S. Lukin , '00

Follow this and additional works at: https://works.swarthmore.edu/fac-physics

Part of the Physics Commons

Let us know how access to these works benefits you

\section{Recommended Citation}

D. A. Schaffner; Michael R. Brown; and Vyacheslav S. Lukin , '00. (2014). "Temporal And Spatial Turbulent Spectra Of MHD Plasma And An Observation Of Variance Anisotropy". Astrophysical Journal. Volume 790, Issue 2. DOI: 10.1088/0004-637X/790/2/126

https://works.swarthmore.edu/fac-physics/111

This work is brought to you for free by Swarthmore College Libraries' Works. It has been accepted for inclusion in Physics \& Astronomy Faculty Works by an authorized administrator of Works. For more information, please contact myworks@swarthmore.edu. 


\title{
TEMPORAL AND SPATIAL TURBULENT SPECTRA OF MHD PLASMA AND AN OBSERVATION OF VARIANCE ANISOTROPY
}

\author{
D. A. SCHAFFNER ${ }^{1}$, M. R. BROWN ${ }^{1}$, AND V. S. LUKIN ${ }^{2}$ \\ ${ }^{1}$ Swarthmore College, Swarthmore, PA, USA \\ ${ }^{2}$ Space Science Division, Naval Research Laboratory, Washington, DC, USA \\ Received 2014 April 14; accepted 2014 June 9; published 2014 July 14
}

\begin{abstract}
The nature of magnetohydrodynamic (MHD) turbulence is analyzed through both temporal and spatial magnetic fluctuation spectra. A magnetically turbulent plasma is produced in the MHD wind tunnel configuration of the Swarthmore Spheromak Experiment. The power of magnetic fluctuations is projected into directions perpendicular and parallel to a local mean field; the ratio of these quantities shows the presence of variance anisotropy which varies as a function of frequency. Comparisons among magnetic, velocity, and density spectra are also made, demonstrating that the energy of the turbulence observed is primarily seeded by magnetic fields created during plasma production. Direct spatial spectra are constructed using multi-channel diagnostics and are used to compare to frequency spectra converted to spatial scales using the Taylor hypothesis. Evidence for the observation of dissipation due to ion inertial length scale physics is also discussed, as well as the role laboratory experiments can play in understanding turbulence typically studied in space settings such as the solar wind. Finally, all turbulence results are shown to compare fairly well to a Hall-MHD simulation of the experiment.
\end{abstract}

Key words: magnetohydrodynamics (MHD) - methods: data analysis - plasmas - solar wind - turbulence

Online-only material: color figures

\section{INTRODUCTION}

As instrumentation capabilities on spacecraft have steadily improved, focus on space plasma turbulence has pushed into ion, sub-ion, and electron-scale regions in both the solar wind (Alexandrova et al. 2009; Sahraoui et al. 2009) and the magnetosheath (Sahraoui et al. 2006; Yordanova et al. 2008). Newer analysis techniques have also been developed to better tap the details of the rich turbulent environments of the heliosphere and magnetosphere.

Analysis of time series fluctuations in the guise of spectrum analysis has served as the foundation for plasma turbulence research, particularly in the solar wind (Goldstein et al. 1995; $\mathrm{Tu} \&$ Marsh 1995). Improving instrumentation has led to ever greater precision in measurements of spectral indices for both magnetic and velocity fluctuation spectra (Podesta et al. 2007), as well as increasing resolution of scale, allowing for measurements of spectra of sub-ion and electron-scale fluctuations (Yordanova et al. 2008; Alexandrova et al. 2009; Sahraoui et al. 2009; Chen et al. 2013). However, many outstanding questions regarding the nature of this turbulence, including both injection and dissipation mechanisms, have prompted research beyond typical spectral analysis. Two such avenues have been the exploration of the anisotropic nature of the turbulence and comparison of spectra between different observables (e.g., magnetic versus density fluctuations).

Theoretical treatments of magnetized plasma turbulence have almost universally predicted anisotropy to develop based on directions perpendicular and parallel to a mean field vector (Montgomery \& Turner 1981; Matthaeus et al. 1990; Goldreich \& Sridhar 1995; Zhou et al. 2004; Boldyrev 2006); many forms of this anisotropy have been predicted and observed (Dasso et al. 2005; Horbury et al. 2008; Podesta 2009; Perri et al. 2009; Wicks et al. 2010; He et al. 2011) - a good overview of the various types and definitions of anisotropy is given in Horbury et al. (2012). Since the solar wind is super-Alfvenic, anisotropy studies of solar wind turbulence generally reference the velocity vector of the bulk flow when defining perpendicular versus parallel fluctuations-typically resulting in a wave number anisotropy measurement. Instead, this paper focuses on variance anisotropy - the unequal ratio of magnetic fluctuation power in components perpendicular versus parallel to the mean magnetic field (Belcher \& Davis 1971; Smith et al. 2006).

Comparison among fluctuation spectra (typically magnetic, flow, and density fluctuations) also provide insight into the nature of the turbulence. For example, relative partition of fluctuation energy between magnetic and kinetic can be useful in making determinations of turbulent properties (Podesta et al. 2007), while comparison of the spectral indices of magnetic and density spectra can provide information about the compressibility of a plasma (e.g., the so-called density bulge Coles \& Harmon 1989; Harmon \& Coles 2005). Comparison of velocity and magnetic spectra at injection scales can also be used to develop hypotheses on the generating mechanisms of the turbulence (Roberts 2010).

As results from space become more detailed, comparisons to simulations and laboratory experiments become more useful in order to better develop the theory as well as inform future space missions. A long gap in laboratory research has occurred since the earliest observations of magnetic turbulence and anisotropy (Robinson \& Rusbridge 1971) as turbulence work in experiments gravitated toward electrostatic turbulence and transport in tokamaks (Liewer 1985; Tynan et al. 2009), but more recently, much progress has been made to develop laboratory experiments that can inform space plasma turbulence research (Howes et al. 2012) as well as begin to make magnetic turbulence measurements (Ren et al. 2011). Experiments conducted in the magnetohydrodynamic (MHD) wind tunnel configuration of the Swarthmore Spheromak Experiment (SSX) have shown the ability to produce and analyze MHD turbulence using many of the same methods (Dudok de Wit et al. 2013) used in the space plasma community, and have produced turbulence measurements that are comparable to in situ results (Schaffner et al. 2014a, 2014b), particularly 
to magnetosheath spectra (Yordanova et al. 2008) and solar wind intermittency (Sorriso-Valvo et al. 1999).

A full spectral analysis of fluctuations in the SSX has been conducted, including magnetic field, density, and Mach flow fluctuations. Using a wavelet transform method to decompose the time series signal, the magnetic field fluctuation spectra can be broken into portions that are parallel or perpendicular to the local magnetic field vector. Analysis of these portions shows that parallel fluctuation power decreases slightly faster than that for perpendicular fluctuations generating a separation in power as a function of increasing frequency. Such a change in variance anisotropy has been observed in solar wind turbulence (Kiyani et al. 2013). The ratio of fluctuation power is shown to grow from nearly isotropic levels in the energy injection scale to a maximum $\perp / \|$ ratio of 3 at frequencies of about $1 \mathrm{MHz}$. Beyond this point, the ratio begins to return to isotropic levels. Though the axial flow speeds of the plasma in the wind tunnel are sub-sonic $\left(M_{z}<1\right)$, if a Taylor hypothesis were to be invoked, this peak in the ratio would correspond to the ion inertial scale length. It is especially noted that for this experiment, the variance anisotropy is observed in a system where, as in the solar wind, the magnetic fields are completely dynamic_-i.e., no large external guide field is present-which is distinct from previous experimental setups (Robinson \& Rusbridge 1971; Ren et al. 2011).

Since density and Mach fluctuation time series are also recorded, comparisons among these spectra are reported. A comparison of Mach number fluctuation spectra (as a proxy for flow) to magnetic spectra in the lowest frequencies suggests that energy injection into the turbulent spectra is primarily magnetic. This result is consistent with the nature of the spheromak generation process. The indices of density and magnetic spectra appear to diverge at a scale consistent with the ion inertial length, where anisotropy is also found to decrease. Both results would be consistent with an increase in compressibility of the plasma due to ion scale physics.

Finally, direct wave number spectra are made using a multichannel probe and are compared to time domain spectra transformed to spatial scales using the Taylor hypothesis. This diagnostic highlights an advantage of laboratory experiments since actual spatial measurements are extremely difficult insitu, though progress is being made using multiple spacecraft (Sahraoui et al. 2006, 2010). The wave number spectra tend to be slightly shallower than their time domain counterparts. Comparison of the wave number spectra with respect to flow gives a hint at an observation of wave number anisotropy, but the results are not as conclusive compared to the variance anisotropy analysis.

All the temporal and spatial spectra analyses are compared to simulations generated using the $\mathrm{HiFi}$ multi-fluid modeling framework (Lukin \& Linton 2011; Leake et al. 2012; Schaffner et al. 2014a). Spectra are generated from a synthetic diagnostic time series of magnetic fields, flow, and density; results compare favorably to the experiment including the observation of variance anisotropy.

The organization of this paper is as follows. First, a brief description of the plasma laboratory is given. Then, an overview of the analysis techniques used to determine perpendicular and parallel fluctuations is provided; details of the method are given in Appendix A. The main results of this paper are provided in Sections 4-6: Section 4 presents the variance anisotropy results, Section 5 compares magnetic fluctuation spectra with those of velocity and density, and Section 6 shows the results of the direct wave number spectra analysis. Section 7 presents the
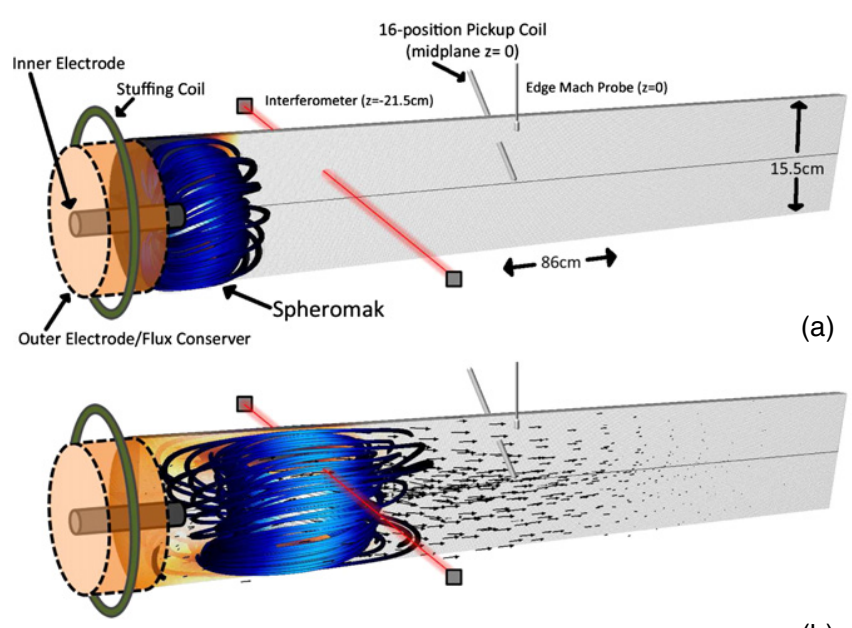

(b)

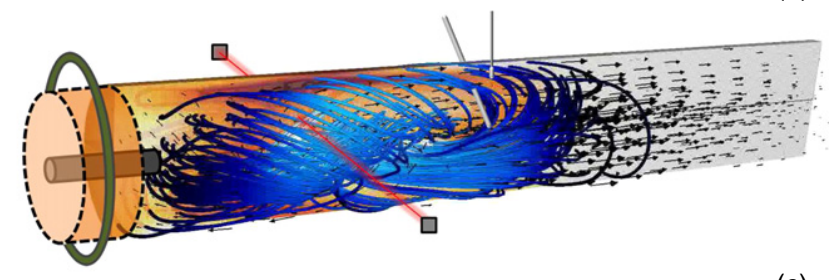

(c)

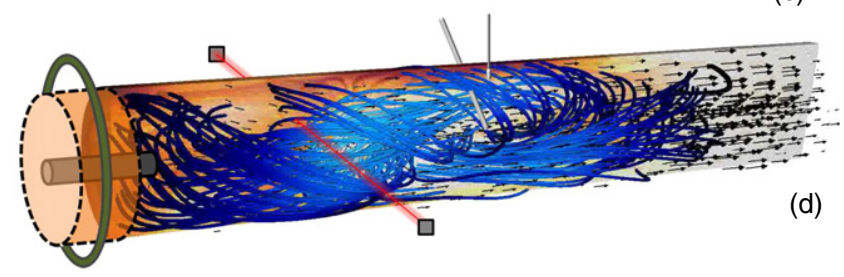

Figure 1. Diagram of the MHD wind tunnel inside the SSX chamber. A plasma gun is located on one end and consists of two electrodes and a stuffing coil. Magnetic pickup coils are located at 16 radial locations at the midplane and a Mach probe measures edge flow at a different angular position at the midplane. The position of the density HeNe interferometer chord is also indicated. Panels (a)-(d) demonstrate the plasma evolution once a spheromak has been formed at the end of the plasma gun (a), and shows the tilt instability (b), twisting under helicity conservation (c) and measurement of turbulent fluctuations (d). The blue lines show simulation generated magnetic field lines, arrows indicate plasma flow magnitude and direction, and orange intensity represents electron density.

(A color version of this figure is available in the online journal.)

comparison of simulations with experimental results. Section 8 discusses the results in light of their relationship to possible ion inertial length scale dissipation. Concluding remarks are given in Section 9. Appendix A gives details of the variance anisotropy analysis, Appendix B shows a comparison of wavelet versus fast Fourier transforms (FFTs), and Appendix C shows a comparison of the anisotropy analysis for local versus global magnetic fields.

\section{EXPERIMENT}

The turbulence injection process in a laboratory experiment is naturally going to have a different origin than a space physics process; however, it is assumed that processes after the energy injection state (i.e., energy transfer in the inertial range and dissipation) will be similar enough so that exploration in the laboratory setting can be beneficial to the understanding of the underlying physical processes.

The energy for the turbulence found in the MHD wind tunnel in the SSX originates in the plasma production process. As diagrammed in Figure 1, a plasma gun configuration sits on 
one end of a $15.5 \mathrm{~cm}$ diameter, $86 \mathrm{~cm}$ long cylindrical copper column that constitutes the MHD wind tunnel. The gun consists of a tungsten-coated $4 \mathrm{~cm}$ diameter inner electrode placed concentrically within the copper cylinder which serves as an outer electrode. An axially aligned wire coil surrounds both electrodes and current is supplied to the coil to produce a known amount of magnetic flux-between 0 and $1.5 \mathrm{mWb}$ - axially through the inner electrode: this flux is referred to as stuffing flux, Ф. A $1 \mathrm{mF}$ capacitor bank, charged to $4 \mathrm{kV}$ is discharged across the electrodes; this voltage fully ionizes a small volume of hydrogen gas puffed in just before the discharge. Radial currents through this newly produced plasma push the plasma down the column and into the fringe magnetic fields which tend to resist this push and stuff the progress of the plasma (hence the term, stuffing flux). Given enough current, and thus large enough $J \times B$ force, the plasma distends the stuffing fields until they break off, forming a self-contained magnetic field structure called a spheromak (Barnes et al. 1986; Jarboe 1994). This structure is visualized in Figure 1(a) using a Hall-MHD simulation generated field lines (in blue). Since the spheromak has both polodial and torodial magnetic fields, the relative ratio of field strength between these two directions is quantified by the magnetic helicity, defined as,

$$
K_{B}=\int A \cdot B d V
$$

where $A$ is the vector potential and $d V$ is the volume element. Previous work on SSX has shown that magnetic helicity of the plasma scales approximately linearly with the amount of stuffing flux applied to the gun (Schaffner et al. 2014b).

Figure 1(a)-(d) illustrates the experimental procedure. The generalized turbulence cascade begins with this compact magnetic structure (Figure 1(a)). Inside the wind tunnel, the magnetic structure is energetically unstable (Bondeson et al. 1981; Jarboe 1994) — the structure will begin to tilt over and expand into the remainder of the wind tunnel (Figure 1(b)). Because the column is copper, and thus flux conserving, the magnetic helicity is conserved unlike the magnetic energy (Taylor 1986); thus, the structure also begins to twist as it tilts over as seen in Figure 1(c). The free energy released in the fall-over materializes as fluctuations in the field, generating the turbulent cascade. The turbulent fluctuations are most prominent in Figure 1(d). In the actual experiment, the gun typically injects more than a single, self-contained structure so while an initial structure is decaying, more compact field energy is being injected (Barnes et al. 1986). This allows for a time frame of stationary fluctuations that is used in the turbulence analysis.

The turbulence data is extracted from magnetic, density, and flow measurements during this stationary period. Magnetic fluctuations are recorded using $3 \mathrm{~mm}$ diameter single loop pickup coils located at 16 radial locations along the radius of the midplane of the column as indicated in Figure 1(a). Each radial position has three orthogonal loops oriented along the axial, radial, and azimuthal directions of the column. A $64 \mathrm{MHz}$, 14 bit DTaq digitizer records $d B / d t=\dot{B}$ time series data which is converted into magnetic fluctuation data in frequency space (as discussed in Section 3). Line-integrated density data are measured with a HeNe interferometer located $21.5 \mathrm{~cm}$ off of the midplane and flow fluctuations are estimated from a Mach probe located on the edge of the copper column at the midplane (as indicated in Figure 1(a)). Spectra of $M_{z}(t)$ are directly reported as a proxy for $V_{z}(t)$, since $\tilde{M} \sim \tilde{V} / C_{s}$ where $C_{s}=\left(T_{e} / m_{i}\right)^{1 / 2}$ and is approximately constant with a
Table 1

MHD Wind Tunnel Plasma Parameters During the Equilibrium Epoch for the Present Configuration of SSX for Non-zero Helicity $\left(K_{B} \neq 0\right)$ and for Zero Helicity $\left(K_{B}=0\right)$ States

\begin{tabular}{lcc}
\hline \hline Parameter & $\begin{array}{c}K_{B} \neq 0 \\
(1.0 \mathrm{mWb})\end{array}$ & $\begin{array}{c}K_{B}=0 \\
(0.0 \mathrm{mWb})\end{array}$ \\
\hline$\langle|B|\rangle(\mathrm{kG})$ & Measured & 0.747 \\
$\langle n\rangle \times 10^{15}\left(\mathrm{~cm}^{-3}\right)$ & 5.283 & 2.84 \\
$\left\langle T_{i}\right\rangle(\mathrm{eV})$ & 1.39 & 17 \\
$V_{\text {bulk }}\left(\mathrm{km} \mathrm{s}^{-1}\right)$ & 23 & 20 \\
\hline & 20 & 5.5 \\
\hline$\beta$ & Computed & 30 \\
$V_{a}\left[\left(\mathrm{~km} \mathrm{~s}{ }^{-1}\right)\right.$ & 0.07 & 31 \\
$C_{s}\left[\left(\mathrm{~km} \mathrm{~s}{ }^{-1}\right)\right.$ & 309 & 0.56 \\
$\rho_{i}(\mathrm{~cm})$ & 31 & 0.43 \\
$\delta_{i}(\mathrm{~cm})$ & 0.09 & 0.05 \\
$\lambda_{\mathrm{mfp}}^{i}(\mathrm{~cm})$ & 0.61 & 1.1 \\
$f_{c i}(\mathrm{MHz})$ & 0.16 & 19 \\
$\nu_{i}(\mathrm{MHz})$ & 8 & 4.3 \\
$f_{\delta i}(\mathrm{MHz})$ & 6 & 3.3 \\
$f_{\rho i}(\mathrm{MHz})$ & 3 & \\
\hline
\end{tabular}

Note. The table has separate sections for directly measured parameters and for quantities computed from these values.

measured value of $T_{e}=10 \mathrm{eV}$ (Zhang et al. 2011; Schaffner et al. 2014b). Bulk flow of the plasma is estimated with timeof-flight measurements between the density signal at $z=$ $-21.5 \mathrm{~cm}$ and the magnetic signal at the midplane, $z=0$. The plasma is also generated with a set amount of magnetic helicity which is governed by initial conditions of the plasma gun source-namely, amount of flux generated in the gun core (the stuffing flux). The helicity can be scanned (Schaffner et al. 2014 b), but in this work, the focus is primarily on two states: a state with non-zero helicity, $K_{B} \neq 0$, generated by $1.0 \mathrm{mWb}$ of flux in the gun core and a state with no injected helicity, $K_{B}=0$. Table 1 indicates typical plasma parameter values for these two states.

Each discharge of the plasma gun constitutes an experimental shot and typically lasts for about $120 \mu$ s from formation of the magnetic fields to their eventual resistive dissipation. Unless otherwise stated, the turbulence data reported here is extracted from a time range of 40-60 $\mu \mathrm{s}$. This is the epoch during each shot where the fluctuations are most stationary; energy at the probe location is balanced between injection energy from the gun and loss through advection away from the probe and through possible dissipation mechanisms (including resistive decay of the currents). An ensemble average for each helicity state is constructed from 40 shots.

\section{ANALYSIS TECHNIQUES}

The bulk of the data of this paper are analyzed in frequency space using either a wavelet transform method (Torrence \& Compo 1998) or a traditional FFT. Time series data are transformed using a sixth-order Morlet mother wavelet with 256 logarithm scale steps per octave. For magnetic fluctuations, the wavelet transform is applied directly to the $\dot{B}_{j}(t)$ data from the pickup coils, separately for each orthogonal component, $j=r, \theta, z$. Each shot contains 8192 time steps and the transform is applied to the entire shot to yield an $W_{j}(f, t)$. A known conversion factor is applied to convert the wavelet scales into 
Fourier frequencies (Torrence \& Compo 1998). The transforms are divided through by the square of the frequency to convert $\dot{B}(f)$ to $B(f)$. Then, the transform is summed over the time range of interest, typically $40-60 \mu \mathrm{s}$, yielding the magnetic fluctuation power,

$$
B_{j}(f)=\frac{1}{f^{2}} \sum_{t=t_{0}}^{t_{1}} W_{j}(f, t)
$$

for a given direction, $j$.

Since the entire shot is being used in the wavelet transform, the frequency range can extend beyond that which would be typical for an FFT given the particular time range under analysis. The entire frequency range is displayed in the following plots, but the focus of the analysis will be on the same frequency range accessible by an FFT. Details on the comparison between the two approaches is given in the Appendix B.

A similar procedure is applied to the density time series from the interferometer and the Mach number time series from the Mach probe. For spatial spectra (using separate radial points on the magnetics probe) an FFT is used.

The method for conversion of the magnetic fluctuation spectra from $\mathbf{B}=\left(B_{r}, B_{\theta}, B_{z}\right)$ to $\mathbf{B}=\left(B_{\|}, B_{\perp}\right)$ for the variance anisotropy analysis utilizes the temporal resolution afforded by the wavelet transformation. A local $\mathbf{B}(t)$-as opposed to a global B (see Appendix C) is determined at each time step, $t$, and the local power spectra for each direction, $B_{j}(f, t)$, is projected onto this magnetic field vector to determine the amount of power perpendicular versus parallel. The projection method is detailed in Appendix A.2 where this method is also compared to other approaches for determining the anisotropy.

\section{VARIANCE ANISOTROPY}

The magnetic field fluctuation power spectra perpendicular and parallel to the local magnetic field vector is shown in Figure 2(a) averaged over 40 shots and the inner four probe tips (a spatial range of $1.5 \mathrm{~cm}$ ). Like previously reported magnetic spectra (Schaffner et al. 2014a), both perpendicular and parallel curves exhibit power-law-like behavior for most frequencies between $10 \mathrm{kHz}$ and $10 \mathrm{MHz}$. Power-law fits to $f^{-\alpha}$ are made to various sections of the curve using a Maximum Likelihood Estimation method (Clauset et al. 2009) —rather than a linear fit to a log-log plot- to help compensate for the small ranges of the fits (Dudok de Wit et al. 2013). The short fits show that the scaling gradually changes from spectral indices of about 1 in the injection (or outer) range to about 5 in the highest frequency sections. As has been noted before, with the exception of the injection range slope, the inertial and possibly dissipation range spectral indices are steeper than observed in solar wind turbulence spectra.

The separation between perpendicular and parallel spectra, however, proceed in a manner similar to that found in space. Both perpendicular fluctuations (red) and parallel fluctuations (blue) begin at roughly the same magnitude in power. Beyond $20 \mathrm{kHz}$, though, the parallel curve dips down slightly faster than the perpendicular curve and this trend continues up to about $500 \mathrm{kHz}$. Then the separation begins to decrease and approaches a constant value from $5 \mathrm{MHz}$ to the Nyquist limit of $32 \mathrm{MHz}$. This gradual change as a function of frequency is more clearly observed in Figure 2(b) plotted in log-linear format, which provides the ratio of the two curves in Figure 2(a). Since the fluctuations perpendicular to the B field have two
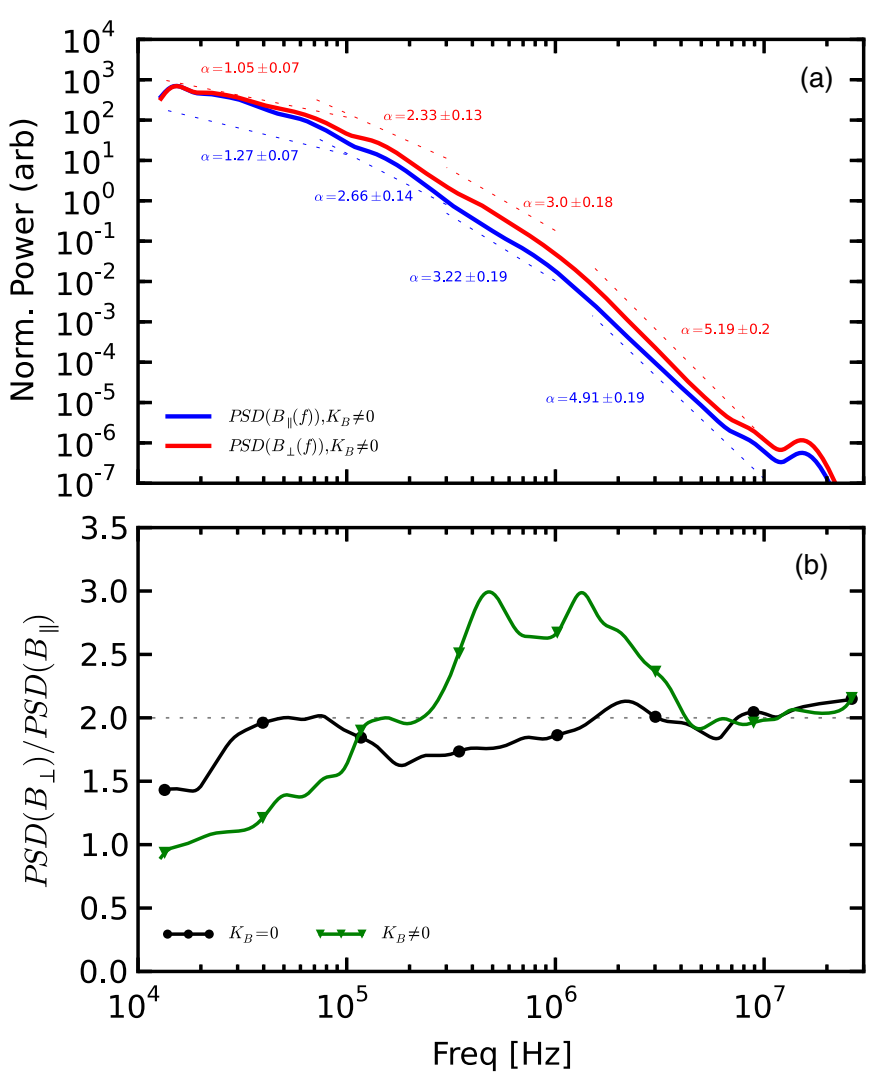

Figure 2. (a) Magnetic power spectra of the non-zero helicity state portioned into perpendicular (red, higher) and parallel (blue, lower) components with respect to the local magnetic field vector direction. Power-law fits and errors are indicated for four sections of the spectra. Values and fit regions are indicated in Table 2. (b) Ratio of the perpendicular over parallel power spectra from (a) as a function of frequency, plotted on a log-linear scale. The green curve (with triangles) shows the $K_{B} \neq 0$ state while the black curve (with circles) shows the result of the same analysis for the zero helicity state. An isotropic ratio is indicated by the horizontal dotted gray line.

(A color version of this figure is available in the online journal.)

component directions while parallel fluctuations have only one, isotropy-equal fluctuation power in all three componentsoccurs when the ratio of perpendicular to parallel is two. Isotropy is indicated in Figure 2(b) by the dashed gray line.

At the lowest frequencies, the balance of power actually tips toward parallel over perpendicular. As the frequency increases, the ratio approaches isotropy, then changes over to dominantly perpendicular power. The ratio continues to steadily increase reaching a peak of about 3 at about $500 \mathrm{kHz}$. There is a brief dip before the ratio rises again at about $1.5 \mathrm{MHz}$; at this point, the ratio begins to drop steadily, and approaches an isotropic level at about $5 \mathrm{MHz}$.

The scale dependency of the $K_{B} \neq 0$ data can be compared to the zero-helicity state. The black curve shows the anisotropy ratio for the zero helicity state, where the field strength is about an order of magnitude less, and there is much less structure to the field. As might be expected for such a state, which has a larger $\beta$ (Smith et al. 2006; Sarkar et al. 2014), there appears to be very little anisotropy at any scale with values ranging close to $R=2$.

Fits to both the spectra and the ratio are shown in Figures 2(a) and 3(a). In Figure 2(a), fits are made to four sections of each curve. The spectral indices, error, and range of fit for both parallel and perpendicular spectra are indicated in the figure and reproduced in Table 2. Overall, comparison of the spectral 

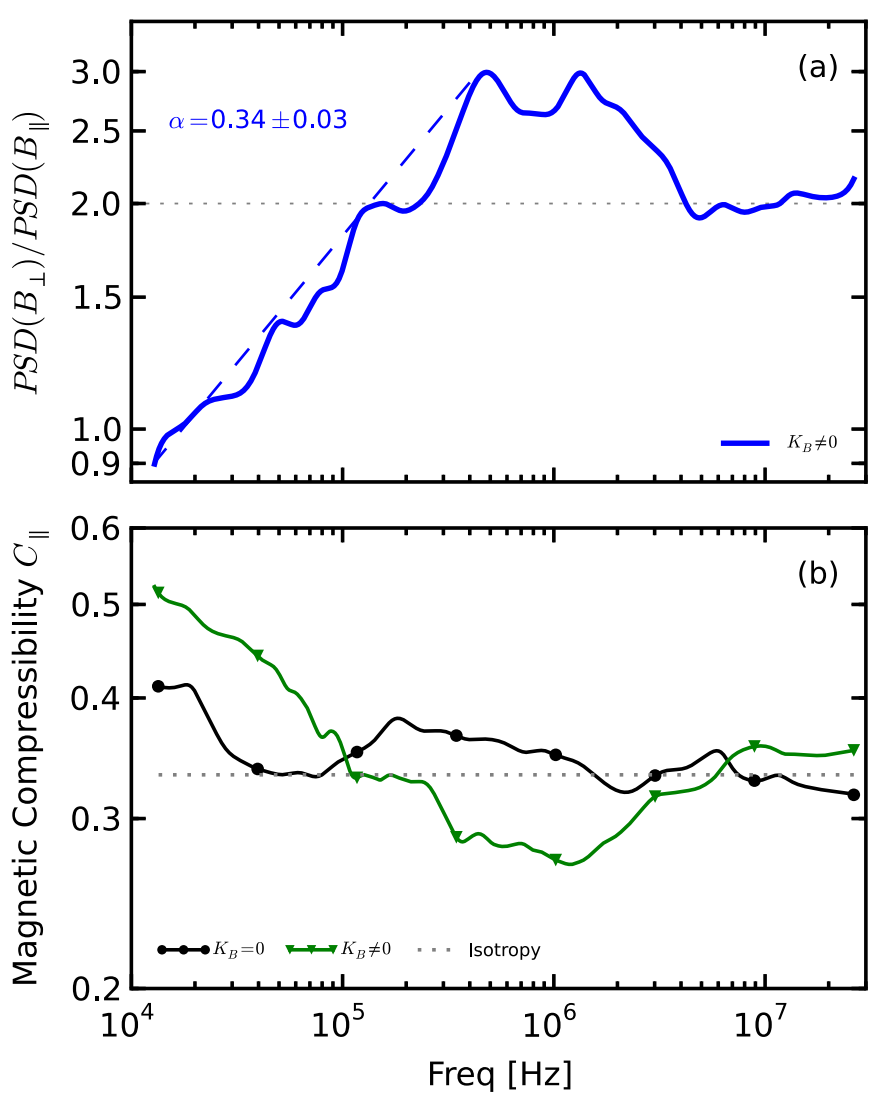

Figure 3. (a) Same quantity as shown with the green curve in Figure 2(b), but now plotted in a log-log format to highlight the power-law behavior as a function of frequency. A fit between $10 \mathrm{kHz}$ and $500 \mathrm{kHz}$ is made with a spectral index of 0.34 indicated above the curve. (b) Magnetic compressibility, defined in Equation (3), for non-zero helicity (green, triangles) and zero helicity (black, circles) as a function of frequency. An isotropic ratio is indicated by the horizontal dotted gray lines in both (a) and (b).

(A color version of this figure is available in the online journal.)

Table 2

Indices from MLE Power-law Fits of Magnetic Fluctuation Spectra in Figure 2

\begin{tabular}{lccc}
\hline \hline $\begin{array}{l}\text { Fit Range } \\
(\mathrm{MHz})\end{array}$ & Direction & Index, $\alpha$ for $f^{-\alpha}$ & Error \\
\hline $0.01-0.1$ & $\|$ & 1.27 & 0.07 \\
& $\perp$ & 1.05 & 0.07 \\
$0.07-0.3$ & $\|$ & 2.66 & 0.14 \\
& $\perp$ & 2.33 & 0.13 \\
$0.3-1.0$ & $\|$ & 3.22 & 0.19 \\
& $\perp$ & 3.00 & 0.18 \\
$1.5-10$ & $\|$ & 4.91 & 0.19 \\
& $\perp$ & 5.19 & 0.20 \\
\hline
\end{tabular}

indicies also reflect anisotropy for frequencies above $1.5 \mathrm{MHz}$; for most fits, the parallel slope is steeper than the perpendicular slope with the exception of the $1.5-10 \mathrm{MHz}$ range. This difference is also reflected in the fit shown in Figure 3(a). An index of $\alpha=0.34$ indicates that the ratio scales approximately as $f^{1 / 3}$ for the region between $10 \mathrm{kHz}$ and $500 \mathrm{kHz}$.

An alternate presentation of this ratio is shown in Figure 3(b), where the ratio of perpendicular and parallel fluctuation power is cast in terms of magnetic compressibility (Kiyani et al. 2013),

$$
C_{\|}(f)=\frac{1}{N} \sum_{i=1}^{N} \frac{B_{\|}\left(t_{i}, f\right)}{B_{\|}\left(t_{i}, f\right)+B_{\perp}\left(t_{i}, f\right)},
$$
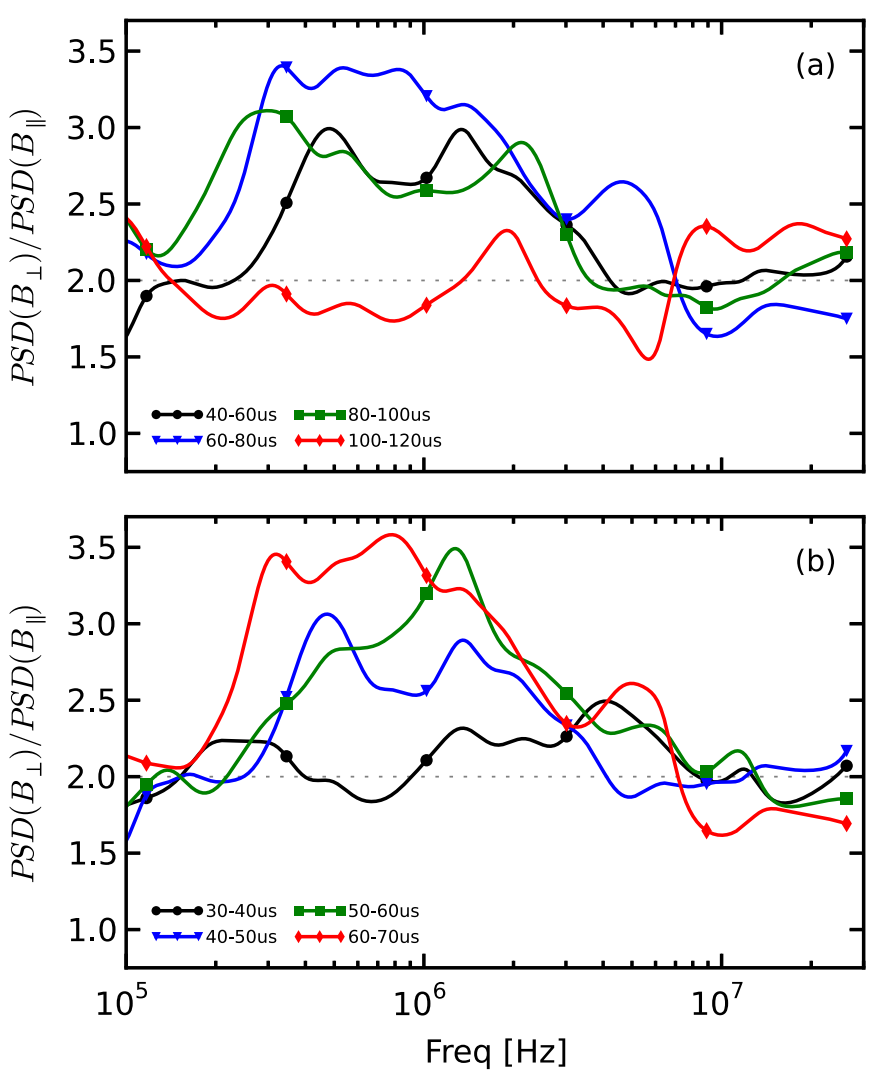

Figure 4. (a) Anisotropy ratio for $K_{B} \neq 0$ data for four $20 \mu$ s time ranges including and following the nominal analysis range of 40-60 $\mu$ s. (b) Anisotropy ratio for $K_{B} \neq 0$ data for four $10 \mu \mathrm{s}$ time ranges just before, including, and just after the nominal analysis range. An isotropic ratio is indicated by the horizontal dotted gray lines.

(A color version of this figure is available in the online journal.)

which relates the anisotropy to the characteristic stiffness of the magnetic field structure. Note the ratio between parallel and total is taken before the wavelet spectra are summed over the time range with this definition while the pure ratio shown in Figure 2(b) and Figure 3(a) is taken after integration over time.

An evolution of the anisotropy over time is also observed. Figure 4 shows the change in the anisotropy ratio as a function of frequency for several time ranges of $20 \mu$ s intervals (Figure 4(a)) and $10 \mu$ s intervals (Figure 4(b)). The black curve in Figure 4(b) shows a period of time, $30-40 \mu \mathrm{s}$, just as the plasma is reaching the midplane probe. The curve remains near isotropic levels for most of the frequencies. For the later time ranges advancing in $10 \mu \mathrm{s}$ intervals, the perpendicular power clearly increases in the $100 \mathrm{kHz}$ to $3 \mathrm{MHz}$ range. The ratio actually peaks in the time frame just beyond the main analysis period shown in Figure 2(b). These trends demonstrate that the anisotropy increases as turbulence evolves. Figure 4(a), shows that after energy is no longer being injected to maintain the turbulence, the anisotropy decreases. After $100 \mu \mathrm{s}$ (red curve), the magnetic field fluctuations have returned to an isotropic ratio.

Some MHD turbulent theories anticipate the ratio of perpendicular fluctuations to parallel fluctuations to increase as a function of decreasing scale size at a particular rate (Goldreich \& Sridhar 1995; Boldyrev 2006). The scaling of $f^{0.34}$ for the ratio of magnetic fluctuation power observed in Figure 3(a) compares very well to the theoretical prediction for the ratio of fluctuation power as a function of wave number rather than frequency, $B_{\perp}(k) / B_{\|}(k) \sim k^{1 / 3}$, but conditionally 

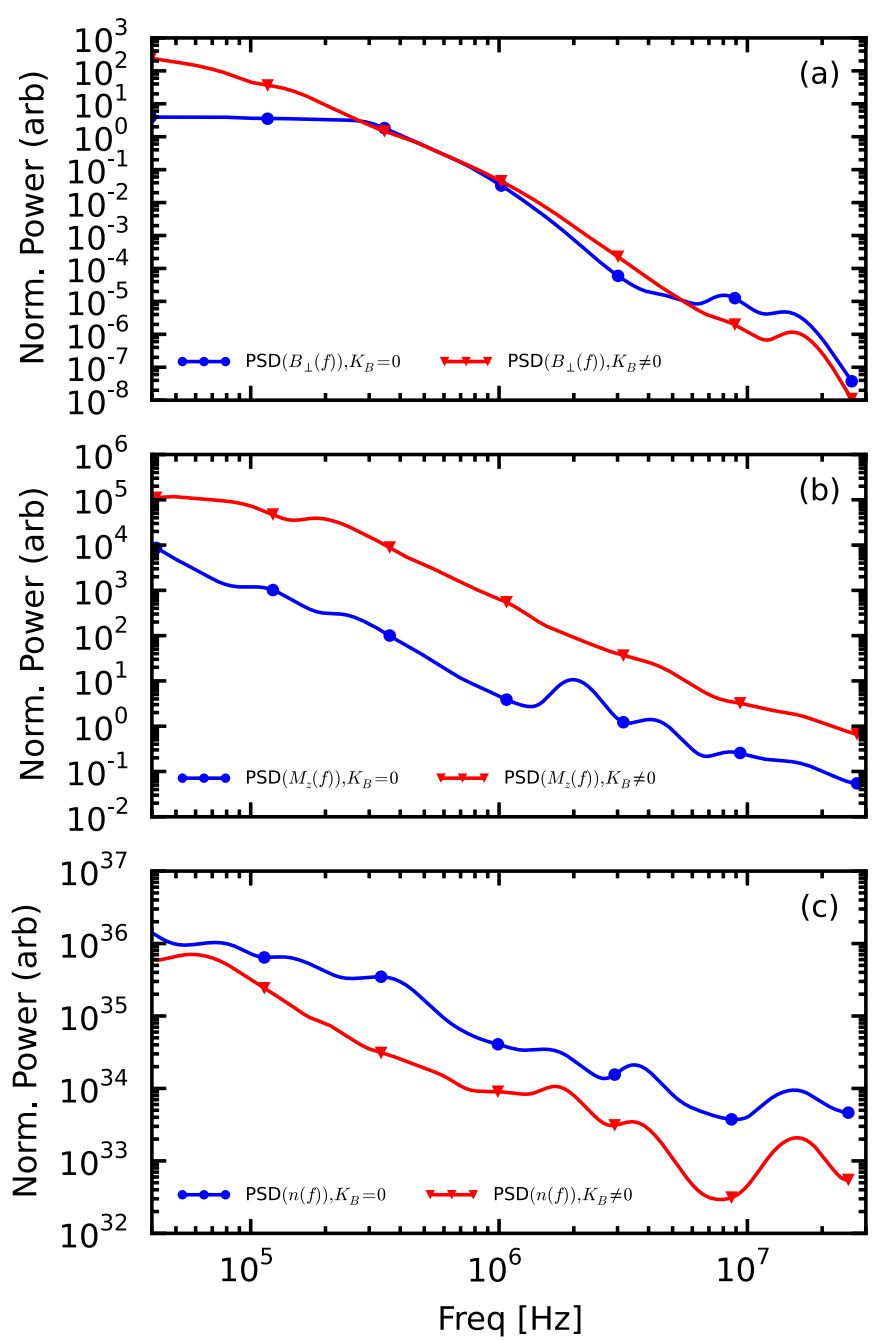

Figure 5. Comparison of spectra between the non-zero helicity $\left(K_{B} \neq 0\right.$, red triangles) and zero helicity ( $K_{B}=0$, blue circles) for (a) magnetic power spectra, (b) Mach number power spectra (as a proxy for velocity spectra), and (c) line-integrated density power spectra. Within each sub-plot, the level of each curve is reflective of the relative fluctuation energy difference between each helicity state.

(A color version of this figure is available in the online journal.)

on the validity of the Taylor hypothesis. The temporal evolution in Figure 4(b) also suggests an increase in anisotropy as the turbulence is given more time to develop. The overall change in anisotropy as a function of frequency (or conversely, the change in magnetic compressibility) can be compared to theories which connect back to the type of fluctuations that may be present in the plasma (Tenbarge et al. 2012; Kiyani et al. 2013). Such theories also motivate the comparison between different parameters (such as magnetic and density fluctuations) which can further illuminate the nature of the fluctuations (Klein et al. 2012) and will be discussed in the following sections.

\section{FLOW AND DENSITY SPECTRA}

For a turbulence cascade to develop, a system needs both energy injection and energy dissipation. The separation of spatial scale between injection and dissipation determines the size of the inertial range. While the actual injection mechanisms of the solar wind are not completely understood, there is evidence from the comparison of large scale magnetic field and velocity fluctuation data that velocity fluctuation energy is being tapped by magnetic fluctuations to sustain an injection-

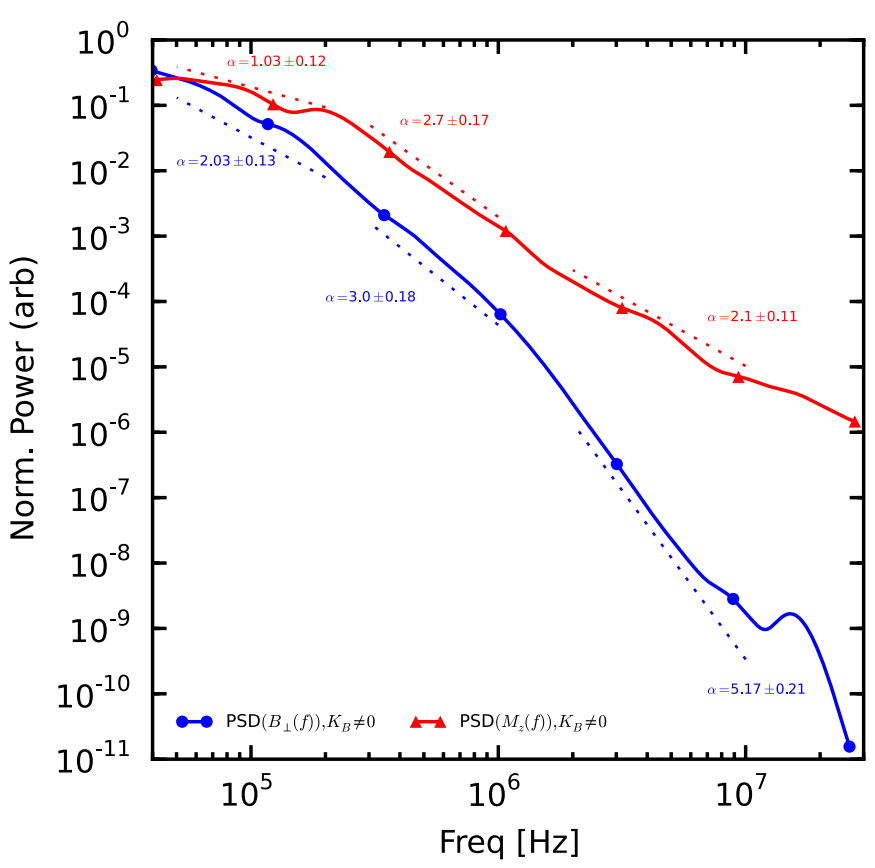

Figure 6. Comparison of magnetic power spectra (blue, circles) and Mach number power spectra (red, triangles) for the non-zero helicity state as a function of frequency. The curves are normalized to their peak values to highlight the comparison of their shapes so relative fluctuation power between the two is not indicated. Fits are made and their values and ranges are indicated in Table 3.

(A color version of this figure is available in the online journal.)

range-like cascade for magnetic spectra (Roberts 2010). In the SSX plasma, however, the injection scale energy is primarily magnetic - the formation of the unstable spheromaks. This is borne out by similar comparisons of magnetic spectra and velocity spectra. Figure 5 shows magnetic spectra (a) and Mach number fluctuation spectra (b) for the two helicity states. As indicated in Table 1, the non-zero and zero helicity states also have different magnetization levels $-5 \mathrm{kG}$ and $0.8 \mathrm{kG}$, respectively.

Comparison of the blue and red curves in Figure 5(a) shows that the larger mean magnetic fields of the non-zero helicity state are correlated with power in low frequency fluctuations while the lower magnetized state has a flat spectrum at these lower frequencies. Comparing these states with the respective velocity fluctuations seems to indicate that the additional magnetic energy injection at larger scales affects the velocity fluctuation power. For the low-field/zero helicity state, the velocity cascade is immediately steep as well as lower in energy overall. In the high-field/non-zero helicity state, however, the velocity fluctuation scaling is shallower, but then has a break point around $200 \mathrm{kHz}$ where the B field fluctuation energy is about the same for the two helicity states. Furthermore, the higher magnetic energy of the $K_{B} \neq 0$ correlates with a higher overall flow fluctuation power of about an order of magnitude. These results suggest that the state with more injected magnetic energy delivers some of its energy to the velocity fluctuations, though more direct evidence of this connection is still being sought.

Figure 6 shows non-zero helicity state magnetic and velocity fluctuation spectra on the same relative scale with power-law fits indicated in the figure and in Table 3. The velocity spectra scales as $f^{-1}$ to about $200 \mathrm{kHz}$, a scaling that is typical for injection range turbulence. Meanwhile, the magnetic spectra is steeper, which implies that some of the magnetic energy may be 
Table 3

Indices from MLE Power-law Fits of Magnetic and Mach Number Fluctuation Spectra in Figure 6

\begin{tabular}{lccc}
\hline $\begin{array}{l}\text { Fit Range } \\
(\mathrm{MHz})\end{array}$ & Parameter & Index, $\alpha$ for $f^{-\alpha}$ & Error \\
\hline $0.05-0.2$ & $M_{z}$ & 1.03 & 0.12 \\
& $B_{\perp}$ & 2.03 & 0.13 \\
$0.3-1.0$ & $M_{z}$ & 2.70 & 0.17 \\
& $B_{\perp}$ & 3.00 & 0.18 \\
$2.0-10$ & $M_{z}$ & 2.10 & 0.11 \\
& $B_{\perp}$ & 5.17 & 0.21 \\
\hline
\end{tabular}

going to drive flows, though the exact mechanism of this energy transfer is not known and its study is reserved for later work.

Beyond $200 \mathrm{kHz}$, the velocity fluctuation scaling steepens, suggesting an inertial range scale. The B field steepens further as well. Then, the curves match slopes for about a decade in frequency space. Beyond $2 \mathrm{MHz}$, the B field scaling drops off significantly while the velocity spectra scaling actually slightly flattens. This is possibly due to a dissipation mechanism that may be further tapping magnetic energy or may be the result of compressive effects (Roberts 2010) of the plasma. Further analysis must be considered before firm conclusions are made as the spectral signal in this data set is approaching the bit-depth limit of the Mach probe digitizer. Alternative velocity fluctuation measurements are also being considered (e.g., electric field fluctuations) for future experimental campaigns.

A comparison of density fluctuation spectra from the line-integrated interferometer time series is also shown in Figure 5(c). Note that the fluctuation power of the $K_{B}=0$ state is higher in this case. The spectra curves of the density tend to be the least power-law-like, so deriving any conclusions from the data at this point is difficult. There is some evidence for a brief rise in the spectra corresponding to ion inertial length scales (about 1-3 MHz) as discussed further in Section 8. Future work will attempt to make a more localized measurement as well as seek correlations between magnetic and density fluctuations which can help to elucidate the underlying nature of the turbulence (Klein et al. 2012).

\section{WAVE NUMBER SPECTRA}

A unique turbulence measurement that can be made in the SSX plasma is a direct wave number spectrum using a multichannel magnetic probe that is inserted radially into the wind tunnel. The probe can measure $\mathbf{B}(t)$ at 16 locations along a $7.3 \mathrm{~cm}$ length of the radius at a spacing of $0.46 \mathrm{~cm}$. In Fourier space, this allows measurements of scales from about 1 to $7 \mathrm{~cm}$. Given that the injection scale of the magnetic energy is on the order of the initial size of the spheromaks- $15.5 \mathrm{~cm}$ - and a dissipation scale can be estimated to be just under $1 \mathrm{~cm}$-ion inertial length for a $1.5 \times 10^{15} \mathrm{~cm}^{-3}$ plasma is on the order of $0.6 \mathrm{~cm}$ - the spatial range sampled by the probe is most likely in the inertial range of the turbulent cascade. Note that $k$ and $1 / \lambda$ (wave number and inverse length) are used interchangeably here, with both intended to mean inverse length scale (i.e., the factor of $2 \pi$ is dropped).

Since the probe can take simultaneous measurements of $\mathbf{B}$ across the plasma, snapshots of the spatial structure of the plasma can be made at each time step. In turn, these spatial distributions can be Fourier transformed to produce power spectra of the scales. Thus, this measurement can capture the direct wave number spectra of the plasma turbulence without
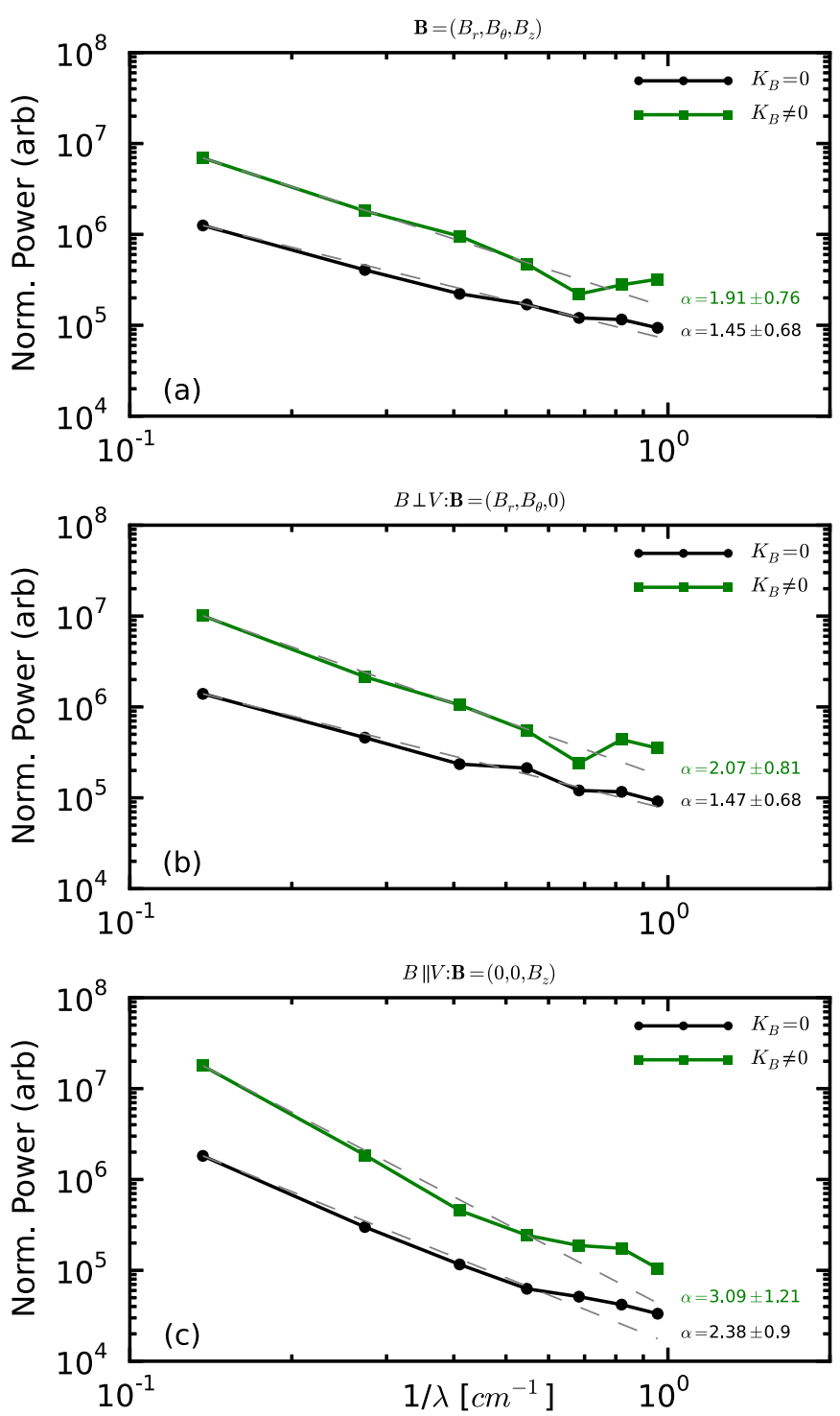

Figure 7. Magnetic wave number power spectra constructed using a multichannel radially oriented probe for both $K_{B} \neq$ (green, squares) and $K_{B}=0$ (black, circles) helicity states. The three panels show this spectrum for (a) the magnitude of the full magnetic field vector, $\mathbf{B}=\left(B_{r}, B_{\theta}, B_{z}\right)$, (b) the magnitude of the projected vector perpendicular to the axial flow, $\mathbf{B}=\left(B_{r}, B_{\theta}, 0\right)$, and (c) the magnitude of the vector parallel to the axial flow, $\mathbf{B}=\left(0,0, B_{z}\right)$. Power-law fits and errors are indicated at the right side of each curve and are fit over the entire data range shown.

(A color version of this figure is available in the online journal.)

reliance on any Doppler shifting as is needed to invoke the Taylor hypothesis. Moreover, since B is constructed from three orthogonal measurements, the power spectra of vectors perpendicular and parallel to the axial flow of the plasma can be separately analyzed.

The downside of the measurement of the wave number spectra in this way is the lack of resolution compared to a frequency spectrum with the assumption of a Taylor hypothesis. With only 16 spatial points, the Fourier spectrum can have only eight points, and only seven can be displayed in log-log format. However, it is a direct measurement-it does not make any physical assumptions - and can be used to cross-reference other observations of spectra.

Figure 7 shows the wave number power spectrum for the two helicity states and for the full vector (a), perpendicular vector (b), and parallel vector (c), averaged for each time step between 


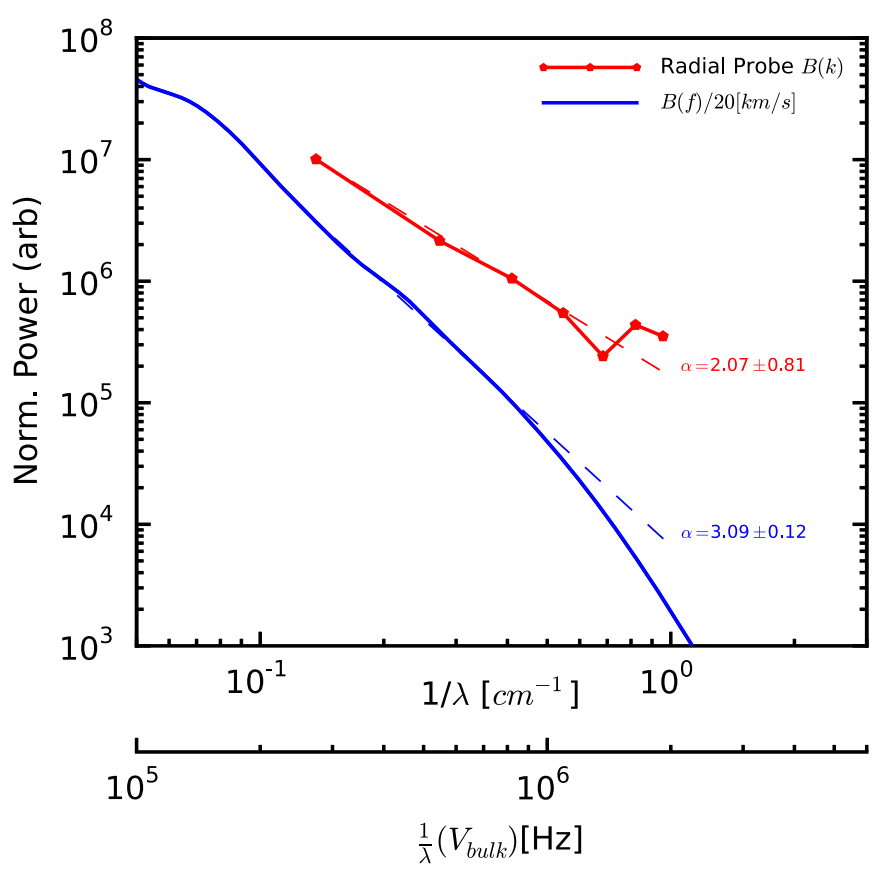

Figure 8. Comparison of a direct wave number spectrum (red, pentagons) and a frequency spectrum (blue) shifted into spatial scales using the Taylor hypothesis and a bulk flow of $20 \mathrm{~km} \mathrm{~s}^{-1}$ as a function of inverse length scale. Fits are made to the same range in inverse length space and are indicated to the right of each curve. The double $x$ axes show the relative correspondence between inverse length scales and frequencies given the Taylor hypothesis shift.

(A color version of this figure is available in the online journal.)

40 and $60 \mu$ s and over 40 shots. Comparison of the curves in Figure 7(a) seems to show a slight variation in the slopes between helicity states, though error bars for the spectral indices are large due to the small number of points usable for the fit. A similar trend is observed between the curves in Figures 7(b) and (c) as well. A larger difference arises when comparing the curves of different vectors. Namely, the separately computed perpendicular and parallel spectra in Figures 7(b) and (c) tend to be slightly steeper than the full vector spectra in Figure 7(a). Moreover, the parallel curves appear to be steeper than the perpendicular curves. The trends are very suggestive of a wave number anisotropy (rather than variance anisotropy). However, there is not as much conclusive evidence for this form of anisotropy as there is for the variance anisotropy.

The wave number spectra and frequency spectra can be directly compared by invoking the Taylor hypothesis for the frequency spectra and Doppler shifting the frequency spectra by the bulk plasma velocity, $V_{p}$,

$$
B(f) \longrightarrow B\left(f-k V_{p}\right) \longrightarrow V_{p} B(k) .
$$

Typically, the last simplification in Equation (4) can only be done if the bulk flow is high enough such that $k V_{p} \gg f$, where $f$ is the temporal frequency of any modes in the plasma. However, in this plasma, the nature of the present modes is still under investigation, so for a first approximation comparison, the full Taylor hypothesis transformation is used. For this plasma, the bulk velocity can be estimated (using both time-of-flight and Mach probe measurements) to be about $20 \mathrm{~km} \mathrm{~s}^{-1}$. The frequency spectra can be plotted on the same scale as the wave number spectra. Figure 8 shows this comparison for the non-zero helicity plasma. The bottom axes show how use of the Taylor hypothesis relates scales of $10 \mathrm{~cm}$ down to $1 \mathrm{~cm}$ to frequencies of $200 \mathrm{kHz}$ to $2 \mathrm{MHz}$. The curves are placed arbitrarily on the $y$ axis. Maximum likelihood estimation powerlaw fits are made to the same range in both curves. The slopes of the curves are comparable suggesting that invoking the Taylor hypothesis for the frequency spectra is not entirely unwarranted. Instead, the steeper slope of the frequency spectra could be reflective of the effect of a combined temporal and spatial scaling, which the direct wave number spectrum does not include. However, breakdown of the Taylor hypothesis has been theoretically predicted to make the spectra shallower rather than steeper (Klein et al. 2014). It also should be noted that these two types of spectra are separately sampling the radial and axial wave number spectra. If the turbulence was completely isotropic, one would not expect a difference between the wave number spectra. Thus, the differences observed might also be reflective of a wave number anisotropy as the direct wave number spectra probes $k_{r}$ and the Doppler-shifted frequency spectra probes $k_{z}$. This possible anisotropy is also hinted at in Figure 7. Future experiments will seek to make a more direct comparison by aligning a multi-channel probe in line with, rather than perpendicular to, the flow, in order to further test the validity of the Taylor hypothesis in this case.

\section{COMPARISON WITH SIMULATIONS}

Simulations of the plasma produced in the SSX wind tunnel have been conducted within the HiFi spectral-element multifluid modeling framework using a set of normalized compressible resistive and Hall-MHD equations. Computational details of the simulations analyzed here have been previously reported (Schaffner et al. 2014a) and have been shown to have favorable comparisons of turbulent spectra and intermittency between simulations and experiments. Further analysis is presented here which shows similar observations of anisotropy, wave number spectra, and velocity B field spectra comparisons to that observed in the experiment. The simulations have parameters most closely resembling the $K_{B} \neq 0$ helicity state.

Time series of quantities in $3 \mathrm{~mm}$ spheres approximately $1 \mathrm{~cm}$ off the central axis and at the midplane are extracted from the simulations for density, three axes of magnetic field, and three axes of velocity. Three axes magnetic fluctuations at 24 radial locations at the midplane are also extracted. To provide some ensemble averaging, points at eight different azimuthal angles are used since only one iteration of the simulation discharge with each of the two MHD models_-resistive and Hall—is presently available.

The simulation time series data are analyzed in a manner similar to the experimental data with the exception that the mother wavelet used for the wavelet transform of the simulation data is a fourth-order Paul rather than a sixth-order Morlet, in order to better capture time resolution for the lesser sampled simulation. A variance anisotropy analysis is also conducted in the same manner, using a local magnetic field and the projection method. Figure 9(a) shows simulation decomposition into perpendicular and parallel spectra compared to $K_{B} \neq 0$ experimental spectra. The simulation data is averaged over eight radially spaced points spanning about $2 \mathrm{~cm}$. The simulation and experimental spectra are staggered on the y axis to emphasize features of the shape, but the perpendicular and parallel curves of each are relative to one another. Clearly, the simulation data exhibit growing variance anisotropy with increasing frequency. The slopes of the simulation spectra match qualitatively well in the region of $100 \mathrm{kHz}$ to $1 \mathrm{MHz}$, though the fit spectra indices indicate a slightly steeper slope than in the experiment (see Table 4). The high frequency end of the simulation spectra drops 

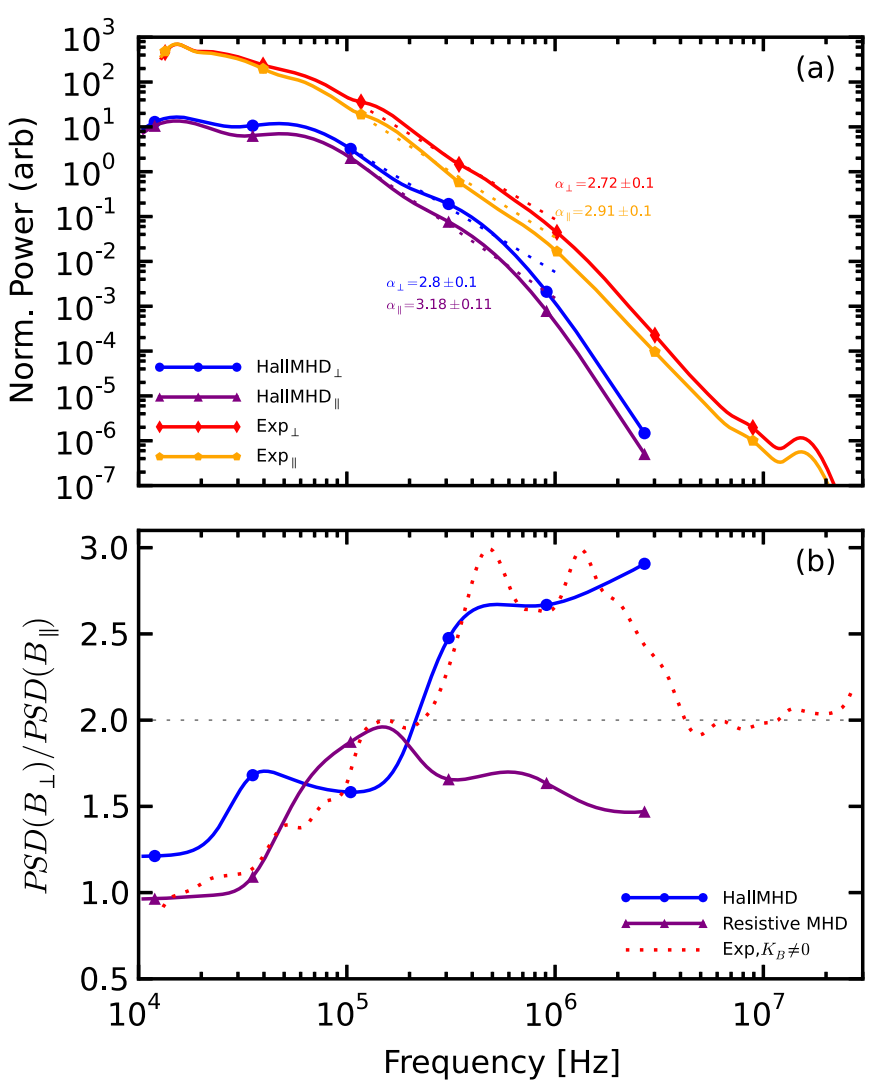

Figure 9. (a) Perpendicular and parallel magnetic power spectra for the simulation (blue circles, purple triangles) and the non-zero helicity experiment (red diamonds, orange pentagons) as a function of frequency. The experimental and simulation curves are arbitrarily placed on the $y$ axis, but the perpendicular (upper) and parallel (lower) curves within each pair are placed relative to each other. Fits and fit regions are indicated in Table 4. (b) Anisotropy ratio as a function of frequency for a Hall-MHD simulation (blue, circles), a resistiveMHD simulation (purple, triangles) and the non-zero helicity experimental data (dotted red). An isotropic ratio is indicated by the horizontal dotted gray line.

(A color version of this figure is available in the online journal.)

Table 4

Indices from MLE Power-law Fits of Magnetic Fluctuation Spectra from the Experiment and Simulation in Figure 9

\begin{tabular}{lccc}
\hline \hline $\begin{array}{l}\text { Fit Range } \\
(\mathrm{MHz})\end{array}$ & Parameter & Index, $\alpha$ for $f^{-\alpha}$ & Error \\
\hline $0.1-1.0$ & $\operatorname{Sim}: B_{\|}$ & 3.22 & 0.11 \\
& $\operatorname{Sim}: B_{\perp}$ & 3.26 & 0.11 \\
& Exp: $B_{\|}$ & 2.91 & 0.10 \\
& Exp: $B_{\perp}$ & 2.72 & 0.10 \\
\hline
\end{tabular}

in power faster than in the experiment, likely due to the limits on sampling time resolution of the simulated data.

The trend in anisotropy ratio is also very similar in the simulation and the experiment, as seen in Figure 9(b). Though the simulation curve does not achieve as large a peak ratio, it does level off at about the same frequency. The clear observation of an increase in ratio suggests that the compressible Hall-MHD physics capture the generation of the anisotropy. Figure 9(b) also shows the anisotropy ratio for a simulation run with the Hall term in the compressible MHD equations set to zero (i.e., resistive MHD). Unlike the Hall MHD and the experiment, the ratio does not switch over to perpendicular dominance, and instead stays near or below the isotropy line. The implications of this have not been analyzed in depth though a Hall term is not

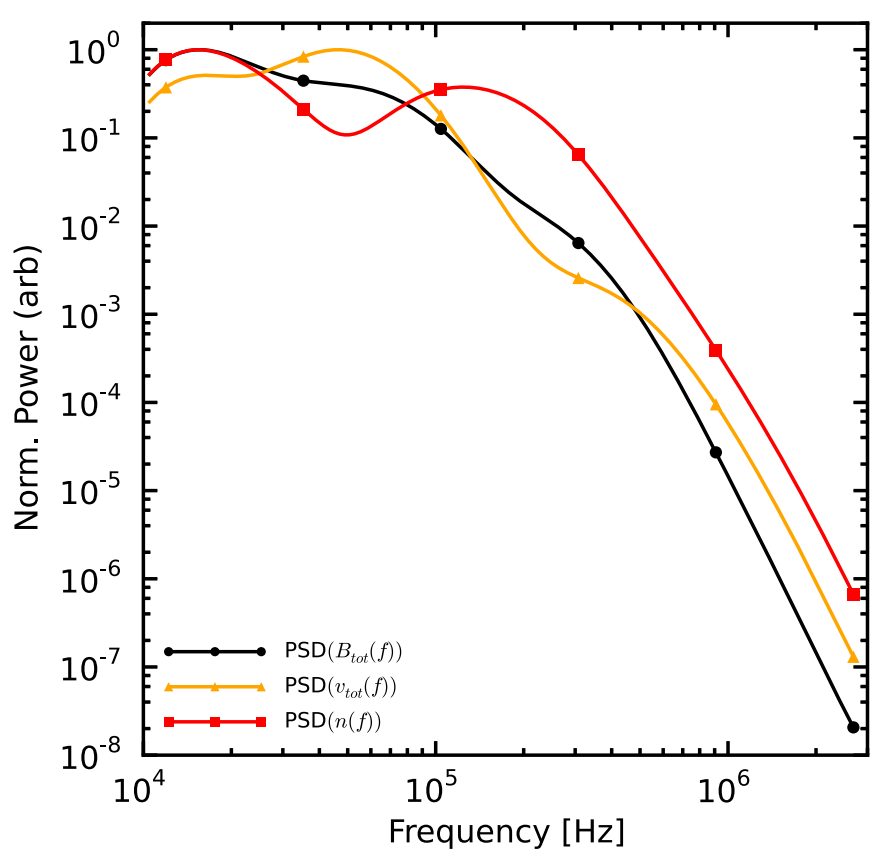

Figure 10. Comparison of simulation-generated total magnetic (black, circles), total velocity (orange, triangles), and density (red, squares) power spectra as a function of frequency.

(A color version of this figure is available in the online journal.)

considered necessary for anisotropy to develop. Also note that the simulation ratio does not decrease again at high frequencies compared to the experiment, but this is again likely due to the lower sampling frequency of the simulated data.

A comparison between velocity and magnetic field fluctuations in the simulation can also be made. Figure 10 shows wavelet-transformed frequency power spectra for the total magnetic field (sum of $B_{r}, B_{\theta}$, and $B_{z}$ ), total velocity, and density, all normalized to their respective peaks. Qualitatively, the comparison between velocity and magnetic field spectra supports the results of the experimental data for non-zero helicity plasmas: the peak in the velocity spectra occurs at a larger frequency than the magnetic spectra. This suggests that energy for the velocity fluctuations are being injected at a smaller scale than the magnetic field fluctuations. Though not conducted here, further analysis of the simulation could potentially show direct energy transfer between the magnetic and velocity fluctuations.

The wave number spectra from a radial cut of the simulation data are also generated using 24 points. Figure 11 shows a direct comparison between simulation and experimental wave number spectra. The slightly higher spatial resolution allows the simulation to reach a smaller scale than the experiment, to about $0.7 \mathrm{~cm}$. In general, the comparison between simulation and experiment is good, suggesting that the simulation is capturing well the spatial structure of the turbulence. Even though the simulation can observe slightly smaller scales, it does not appear to probe small enough scales to exhibit any dissipation effects at ion inertial length scales, which for the simulation is at about $0.7 \mathrm{~cm}$.

As in the experiment, the spatial and temporal spectra of the simulation is compared and shown in Figure 12. The simulation has a bulk axial flow of $18 \mathrm{~km} \mathrm{~s}^{-1}$, close to the $20 \mathrm{~km} \mathrm{~s}^{-1}$ observed in the experiment. A similar trend is seen with the spatial spectra having a shallower slope than the Doppler-shifted frequency spectra. The main difference again appears to be that the frequency spectra hit the limits of the temporal resolution at lower frequencies than the experiment spectra. 

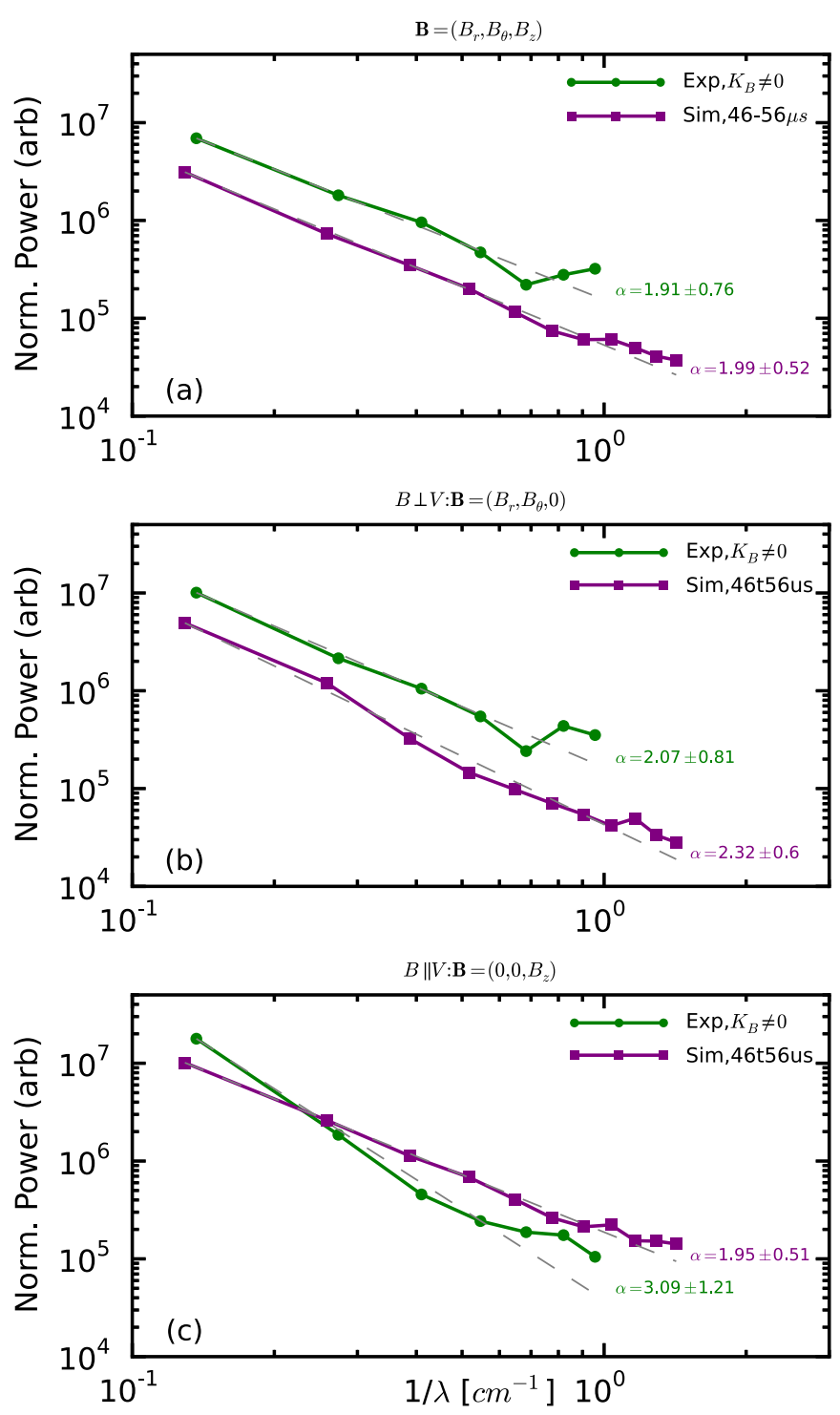

Figure 11. Direct magnetic wave number power spectra from the non-zero helicity data and the Hall-MHD simulation. The three sections indicate the same vector choices described in the caption of Figure 7. Fits to each are displayed to the right of each curve and comprise the entire data range shown for each respective curve. The simulation probes slightly smaller spatial scales since 24 points are used to span the same spatial range rather than 16 points for the experimental probe.

(A color version of this figure is available in the online journal.)

These further comparisons of turbulent statistics and characteristics between the experimental plasma and a compressible Hall-MHD simulation help validate the model as useful for understanding the physical processes. Subsequent simulation analysis will entail more detailed computation of how energy is being distributed and moved through the plasma including relationships between magnetic field and velocity as well as between magnetic fluctuations perpendicular and parallel to a local B field. Higher time resolutions to probe ion scale physics and the effects of varying helicity will also be explored.

\section{DISCUSSION AND EVIDENCE FOR ION SCALE EFFECTS}

A major remaining question for this analysis is whether the plasma diagnostics are able to observe effects of a dissipation scale in this turbulence. Perhaps a more general question can

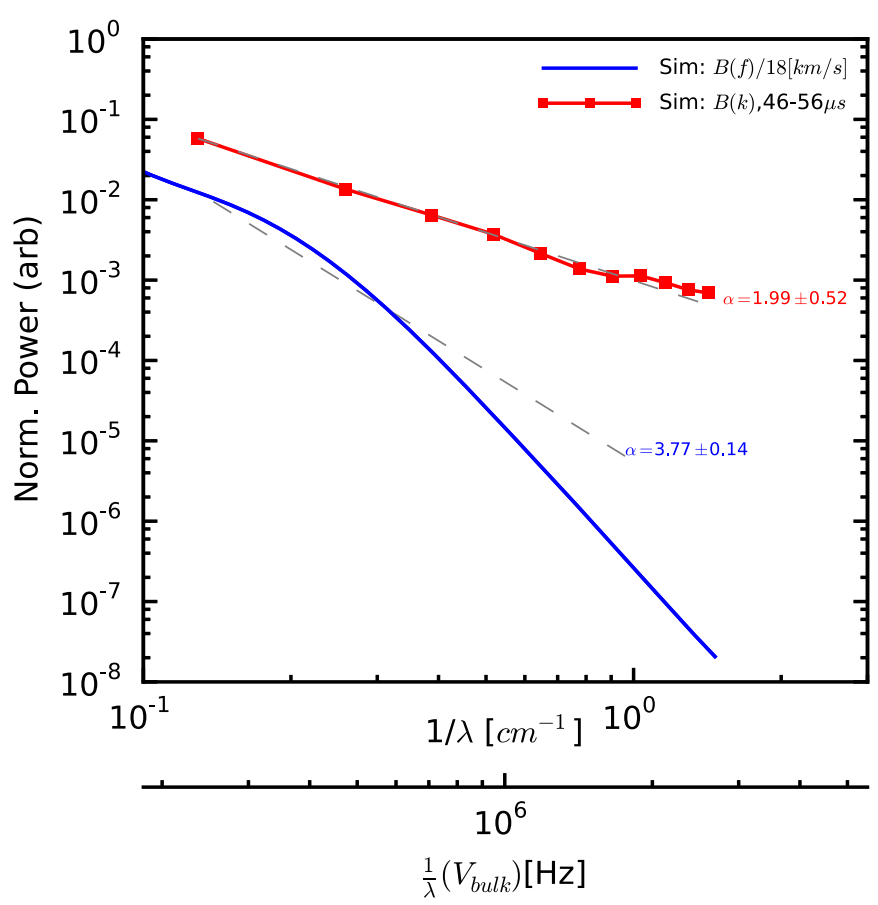

Figure 12. Similar comparison of wave number and converted frequency spectra for simulation data as made in Figure 8, though for the simulation, the bulk flow is $18 \mathrm{~km} \mathrm{~s}^{-1}$ so the correspondence between the two $x$ axis scales is slightly different than in the previous plot.

(A color version of this figure is available in the online journal.)

be posed: How well does this plasma exhibit a traditional fluidturbulence-like picture which posits an injection scale, inertial scale, and dissipation scale?

The results of this analysis provide a number of hints that the ion inertial scale length is being probed, but no one piece of evidence is strong enough to make a conclusive assertion. The first clue arises by comparing the break point of the magnetic spectra with the Doppler-shifted ion inertial scale length, $f_{\delta i}$. Figure 13(a) shows the spectra for magnetic field, Mach number, and density for the $K_{B} \neq 0$ data all normalized to their respective maximum values, with dashed lines indicating the Doppler-shifted frequency of ion inertial length, $f_{\delta i}$, using a bulk flow speed of $20 \mathrm{~km} \mathrm{~s}^{-1}$, the collision frequency, $v_{i}$, and ion cyclotron frequency, $f_{c i}$. Note that the break point occurs just before the ion inertial frequency is reached. Since the ion inertial scale is often associated with the scale size of reconnection layers or current sheets, a break point just preceding this scale suggests the onset of a dissipation mechanism associated with current sheets of some form.

Supporting pieces of evidence for this hypothesis come from the comparison of the density, flow, and magnetic spectra; a comparison to trends in variance anisotropy; and a comparison to observations in space plasmas. The red curve in Figure 13(a) shows a slight bump around $f_{\delta i}$. This is possibly evidence of the density bump effect observed when the plasma becomes more compressible-which would be expected at ion inertial length scales. The flattening of the velocity spectrum has also been speculated to be a signal of compressive effects (Roberts 2010), but no clear-cut relationship has as yet been observed. Similarly, the anisotropy ratio in Figure 2(b) begins to decrease at about this frequency (see Figure 3) which also suggests an increase in compressibility. Such decreases in the anisotropy ratio at dissipation scales have been observed in the solar wind (Hamilton et al. 2008; Kiyani et al. 2013). The spectral slopes 

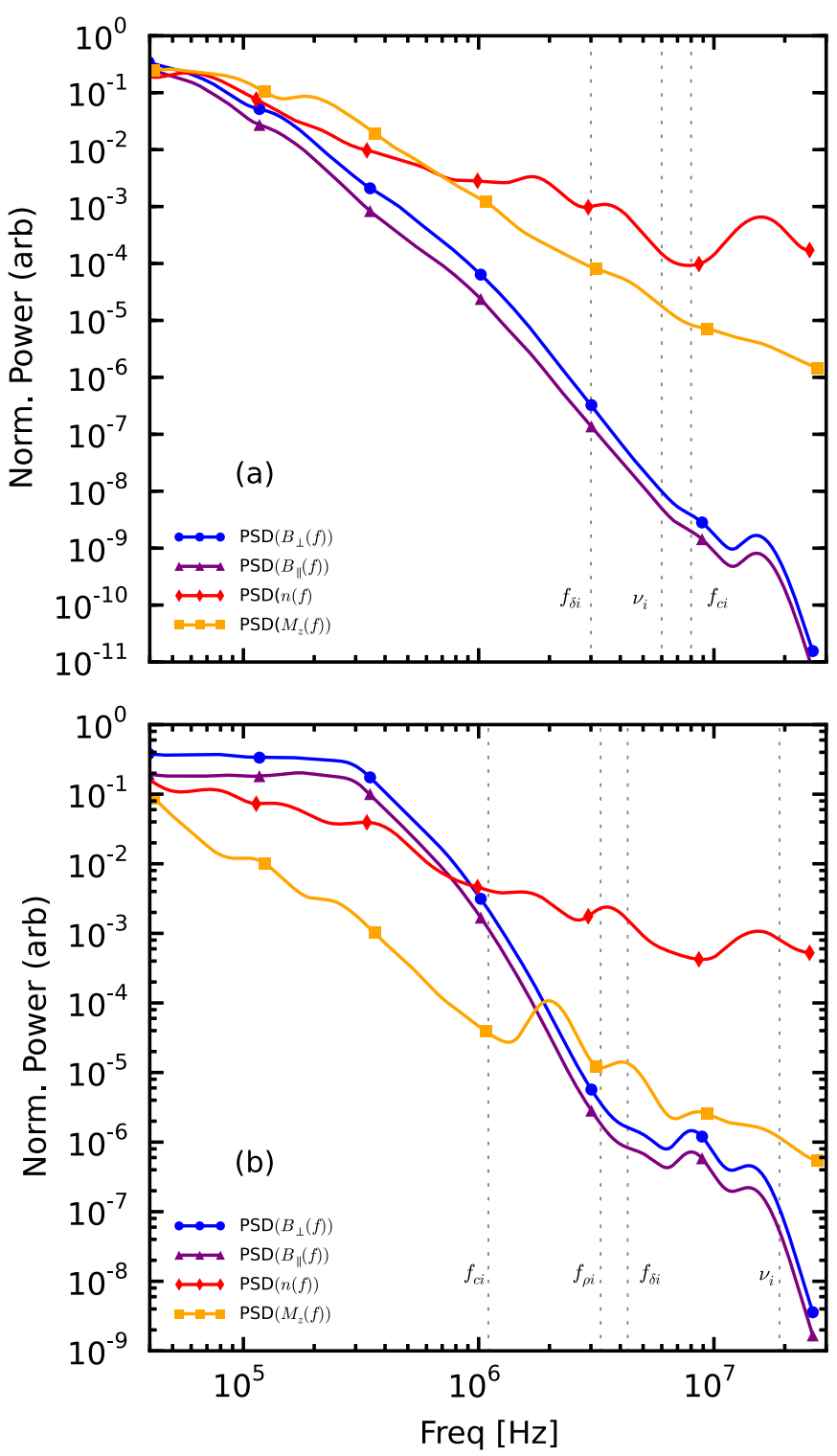

Figure 13. (a) Comparison of magnetic (perpendicular, blue circles; parallel, purple triangles), Mach (orange squares), and density (red diamonds) spectra all normalized to their respective maximum value to highlight differences in spectral shape for $K_{B} \neq 0$ data. Vertical dashed lines indicate the position in frequency space of the Doppler-shifted ion inertial length, $f_{\delta i}$, the collision frequency, $v_{i}$, and the ion cyclotron frequency, $f_{c i}$. (b) Similar comparison as (a), but for the zero helicity case. The Doppler-shifted ion gyroradius, $f_{\rho i}$ is also indicated on this second subplot.

(A color version of this figure is available in the online journal.)

and break point at $c / \omega_{p i}$ of the magnetic spectrum also compare well with observations made in the magnetosheath (Yordanova et al. 2008).

However, other explanations for these effects remain. One, the Taylor hypothesis used to establish the connection between the frequency and the scale length here is not, as yet, conclusively applicable. If the Taylor hypothesis cannot be invoked, there may be other reasons for the observed break point. Also, the flattening of the spectra in Figure 13(a) may be due to reaching the noise floor set by the bit-depth of the interferometer/Mach probe data acquisition system (which is smaller than the 14 bit range of the magnetic data). Moreover, there is evidence, at least in the solar wind, that density spectra might be expected to steepen, not flatten, beyond ion scales (Chen et al. 2012), not flatten.
Comparison of the two different helicity states only adds to the ambiguity. Figure 13(b) shows the same curves as in (a), but for the zero helicity state. The break point in the magnetic field here appears to occur close to the ion cyclotron frequency rather than the ion inertial length. It should be noted though that previous work (Schaffner et al. 2014b) has suggested that the zero helicity state consists of much fewer current sheets and as such, dissipation in this state would depend much less on those mechanisms. The zero helicity state also shows no change in anisotropy with scale and no density bump.

The results presented nevertheless highlight the need for further investigation, particularly into whether a Taylor hypothesis can be invoked, or at the very least, whether some type of Doppler shift can be applied to properly connect the frequency and scale size of the signal. Similarly, a higher resolution in the spatial probes could provide confirmation as its current incarnation just misses the apparent dissipation scale. Moreover, better resolved density and Mach flow diagnostics would be useful in order to distinguish between noise and an actual effect.

Lastly, simulations could potentially provide further insight into the processes occurring. Unfortunately, the comparison with the presently available simulation diverges at scales associated with ion physics. This indicates the need to re-examine the simulation at higher temporal sampling rates, but could also reflect the need to include other terms such as ion finite Larmour radius effects or gyroviscosity in order to better reproduce the correct dissipation physics.

\section{CONCLUSIONS}

This manuscript presents a broad analysis of both temporal and spatial fluctuation spectra with the intent of understanding the MHD turbulence observed in a wind tunnel on the SSX. The results show the presence of variance anisotropy-more power in fluctuations perpendicular to the local magnetic field than parallel to it - for a plasma with non-zero injected helicity. The ratio of perpendicular to parallel magnetic fluctuation power shows variation as a function of frequency and of time, reaching peak values of about $R=3$ during the period of mostly stationary fluctuations and at frequencies between $500 \mathrm{kHz}$ and $1 \mathrm{MHz}$. Very little anisotropy is observed for a zero helicity state, which may be more closely related to the value of $\beta$ for this state of the plasma rather than to the helicity itself. Furthermore, this variance anisotropy is observed in a system that does not have a large external guide field.

Comparison of fluctuation spectra of magnetic fields and velocity appear to support the notion that energy is primarily injected into the plasma through magnetic fields (during the spheromak formation process). Comparison among magnetic, velocity, and density spectra provide clues for the nature of the dissipation processes occurring in the plasma, but much more research is needed for definitive conclusions to be made.

A direct wave number spectrum is also measured in the plasma, highlighting a possible advantage that measurements in laboratory plasmas can have over in situ space measurements. The wave number spectra show slight differences when compared to temporal frequency spectra converted into spatial scales using the Taylor hypothesis. The measured bulk axial flow of the plasma wave number spectra also suggest a wave number anisotropy, but the results are less conclusive than the observation of the variance anisotropy. These initial results, however, provide the impetus for more detailed spatial turbulent measurements in future experimental campaigns including the 
possibility of testing the validity of the Taylor hypothesis in this plasma.

All of these results are also compared to a Hall-MHD computation generated to simulate the spheromak relaxation process. Turbulent spectra using synthetic diagnostics compare favorably to the experiment particularly in the measured spectral indices for power-law fits, but comparisons tend to diverge at higher frequencies where more ion scale effects may be in play. Improved data extraction techniques are being sought in order to produce higher temporally resolved simulation data.

Many open questions remain though, including whether or not dissipation at the ion inertial length scale is observed. Both the magnetic spectra break point and a decrease in anisotropy occur at scales associated with the ion inertial length, but only under the assumption of the Taylor hypothesis. The direct wave number spectra do not probe a small enough scale to provide any evidence for or against this hypothesis. The encouraging comparisons with simulations, though, might provide some support if they can be pushed to higher resolutions. Other diagnostic techniques are also being pursued to help unravel this issue.

The goal of this analysis is to establish the use of the MHD wind tunnel as a testbed for understanding turbulence typically researched at space physics scales. Many of these results have intriguing comparisons with turbulent situations in the solar wind and the magnetosheath-both for cases where the data compares well, as well as cases where it does not. The advantage of laboratory experiments lies in the ability to more easily make spatial measurements, the ability to have some nominal control over parameters, and the ability to make many repeatable measurements. Through a combination of these laboratory observations, computer simulations, and space observations, a greater understanding of MHD turbulence is sought.

We gratefully acknowledge many useful discussions with William Matthaeus, Greg Howes, Kris Klein, Robert Wicks, Jason TenBarge, and Adrian Wan. This work has been funded by DOE OFES and NSF CMSO. The simulations were performed using the advanced computing resources (Cray XC30 Edison system) at the National Energy Research Scientific Computing Center.

\section{APPENDIX A}

\section{COMPUTATION OF VARIANCE ANISOTROPY}

The computation of the variance anisotropy involves two main steps: a determination of fluctuation power and an estimate of the distribution of the fluctuation power relative to a vector direction. The first step is accomplished using a wavelet transform procedure as is discussed in the text. The division of fluctuation power is accomplished using what will be described here as a projection method. A second estimate of fluctuation power distribution is constructed using a threshold method and is also described in detail in this Appendix. The threshold method is a more straightforward process for determining the level of variance anisotropy, but suffers from diminishing statistics. Its presentation here is mainly as a validation of the eventual use of the projection method for the analysis in the paper.

\section{A.1. Threshold Method}

Both the threshold method and the projection method for determining variance anisotropy rely on the ability of the wavelet transform to yield a power spectrum distribution as a function of both time and frequency (i.e., $B(f, t)$ ). A local (in time) vector $\mathbf{B}$ is determined for each time, $t$,

$$
\mathbf{B}(t)=B_{r}(t) \hat{r}+B_{\theta}(t) \hat{\theta}+B_{z}(t) \hat{z}
$$

where each component $j=r, \theta, z$ is determined from $\dot{B}_{j}$ by integrating over time as

$$
B_{j}(t)=\int_{0}^{t} \frac{d}{d \tau}\left(B_{j}(\tau)\right) d \tau .
$$

Since the magnetic probe measures orthogonal magnetic field directions by construction, this fact can be used to directly seek a difference in fluctuation spectra depending on orientation perpendicular or parallel to the overall field. Thus, a threshold ratio for each component can be defined as a fraction of the total magnitude as in,

$$
R_{j} \geqslant \frac{\left|B_{j}\right|^{2}}{|\mathbf{B}|^{2}},
$$

which reflects the relative amount that the total vector points in one of the three orthogonal directions. Then for every time, $t$, in a given time range and for each shot, the quantity $B_{j}(f, t)$ is summed for each $t$ where

$$
\frac{\left|B_{j}\right|^{2}}{|\mathbf{B}|^{2}} \geqslant R_{j}
$$

for all frequencies, $f$. The value chosen for $R_{j}$ determines how strictly the total vector aligns with the component, $j$. By definition, then, the summed power for $B_{j}$ is considered the parallel component and the sum of the remaining two directions is the perpendicular component. If there is any anisotropy in the signal, a difference in energy content of the spectra should become apparent as the threshold value is increased.

Indeed, an effect like this is observed. Figure 14 shows the ratio of total perpendicular fluctuation power to parallel fluctuation power for $j=r$ (Figure 14(a)) and $j=\theta$ (Figure 14(b)). The threshold fraction is indicated by color. The dashed line at two represents isotropy-where the sum of two perpendicular components is about twice the power of the single parallel component. Clearly, for the lowest threshold value, the ratio remains close to the isotropy line for all frequencies as would be expected. As the threshold value is raised, the ratio from about $10 \mathrm{kHz}$ and higher begins to grow. This shows there is variance anisotropy in the plasma. If the plasma was isotropic, a difference between perpendicular and parallel spectra would not be seen. The anisotropy ratio reaches a maximum as the threshold nears $100 \%$. The drawback to this method, however, is that as the threshold is increased, the number of individual spectra summed is reduced, which increases the error of each curve. This also probably explains why the threshold method indicates higher ratios than the more highly averaged data from the projection method discussed next.

\section{A.2. Projection Method}

An alternative method, and the one that is used to compute the results presented in this manuscript, uses the $\mathbf{B}$ time series data to project spectral power into perpendicular and parallel portions at each time step. This projection method uses all the available time steps and shots rather than making a cut like the threshold method. It will be shown later that the two methods 

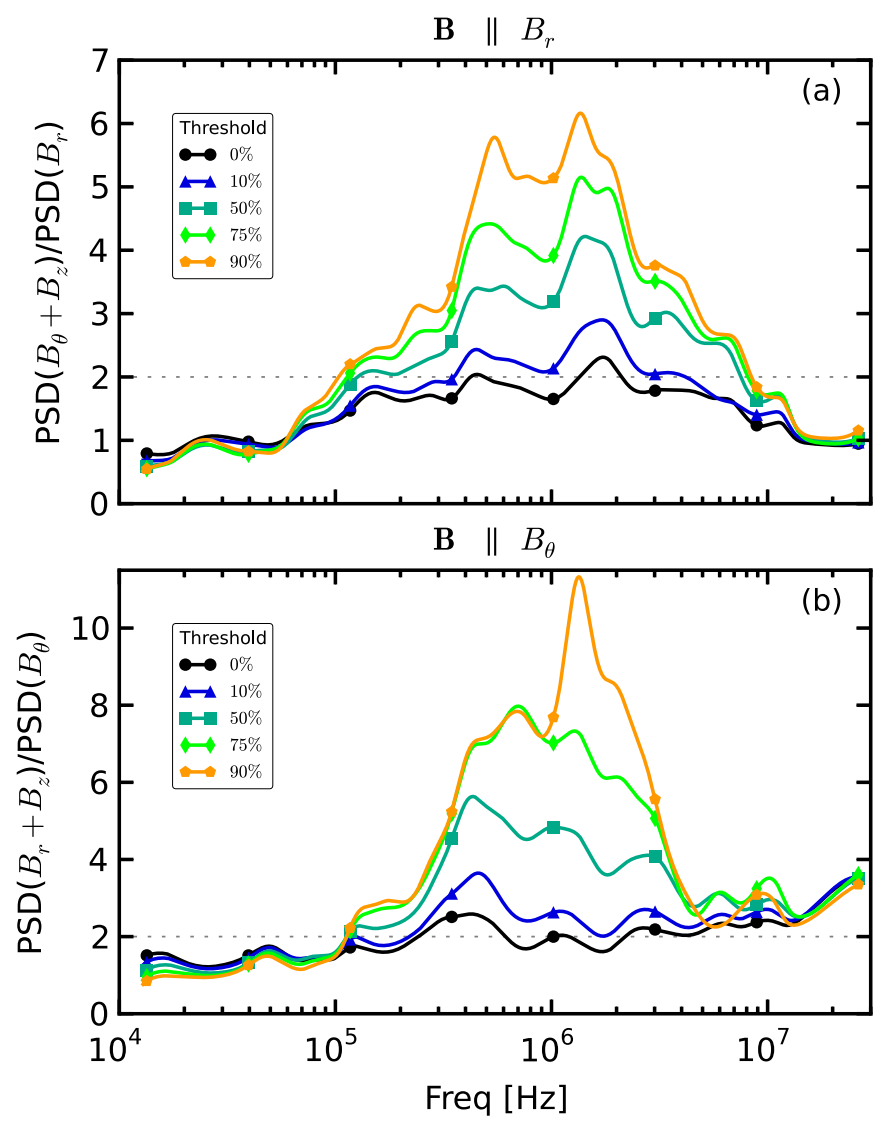

Figure 14. (a) Ratio of the sum of magnetic power spectra of $B_{\theta}$ and $B_{z}$ over the spectra of $B_{r}$ as a function of frequency and summed over the number of instances in time when the threshold ratio, defined in Equation (A3) for $j=r$, is surpassed. Each curve indicates the level of anisotropy for different threshold values. The threshold ratio indicates the relative amount the full magnetic field vector, B points in the direction of $B_{r}$. (b) Similar to (a), but for $j=\theta$.

(A color version of this figure is available in the online journal.)

give quantitatively similar answers for the amount of variance anisotropy.

The projection method also uses the wavelet transform to compute $B_{j}(f, t)$. However, rather than use the $\mathbf{B}(t)$ as a threshold value, it is used as a reference vector to determine what fraction of the fluctuation power of $B_{r}(f, t), B_{\theta}(f, t)$, and $B_{z}(f, t)$ is perpendicular or parallel to that vector. The parallel component of each $B_{j}(f, t)$ is found by computing the projection,

$$
\operatorname{Proj}_{u} v=\frac{\mathbf{v} \cdot \mathbf{u}}{\|\mathbf{u}\|} \mathbf{u},
$$

which shows that the magnitude of the component of $B_{j}$ parallel to $\mathbf{B}$ is

$$
\left(B_{j}^{\|}\right)^{2}=\frac{B_{j}^{2}}{|\mathbf{B}|^{2}} .
$$

Then, the magnitude of the component perpendicular is

$$
\left(B_{j}^{\perp}\right)^{2}=1-\frac{B_{j}^{2}}{|\mathbf{B}|^{2}} \text {. }
$$

Using these projection coefficients, each wavelet transform spectra, $B_{j}(f, t)$ for $j=r, \theta, z$, can be divided into $B^{\|}(f, t)$ and $B^{\perp}(f, t)$. For each time step during each shot, the total parallel and perpendicular power is found by summing the respective
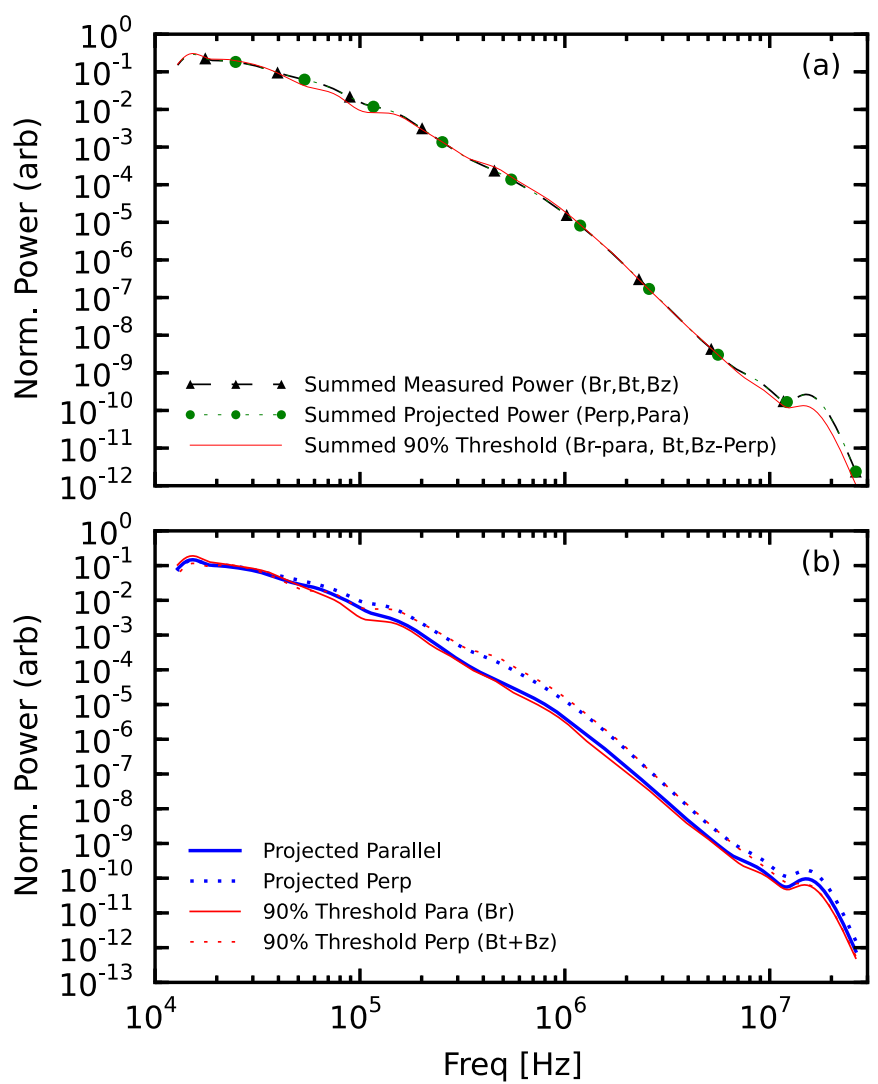

Figure 15. (a) Comparison of the total summed magnetic power showing the exact correspondence between $\left(B_{r}, B_{\theta}, B z\right)$ and $\left(B_{\|}, B_{\perp}\right)$. The red curve shows the total power for the $90 \%$ threshold level which, while not exact, is reasonably close to the other two curves. (b) Comparison of anisotropy in magnetic spectra using the projected method and the threshold method.

(A color version of this figure is available in the online journal.)

portions from each orthogonal direction, as in

$$
\begin{aligned}
B_{\|}(f)= & \sum_{t=t_{0}}^{t_{n}}\left(B_{r}^{\|}\right)^{2}\left(B_{r}(f, t)\right) \\
& +\left(B_{\theta}^{\|}\right)^{2}\left(B_{\theta}(f, t)\right)+\left(B_{z}^{\|}\right)^{2}\left(B_{z}(f, t)\right) \\
B_{\perp}(f)= & \sum_{t=t_{0}}^{t_{n}}\left(B_{r}^{\perp}\right)^{2}\left(B_{r}(f, t)\right) \\
& +\left(B_{\theta}^{\perp}\right)^{2}\left(B_{\theta}(f, t)\right)+\left(B_{z}^{\perp}\right)^{2}\left(B_{z}(f, t)\right)
\end{aligned}
$$

for the given time range $t_{0} \leqslant t \leqslant t_{n}$. These summed quantities, $B^{\|}(f, t)$ and $B^{\perp}(f, t)$, are what are used in Section 4.

As a check, the total fluctuation power spectrum is computed in three different ways and plotted in Figure 15(a) for a time range of 40-60 $\mu \mathrm{s}$. The total power is found by (1) summing $B_{r}(f), B_{\theta}(f)$, and $B_{z}(f)$ directly, (2) summing $B^{\|}(f)$ and $B^{\perp}(f),(3)$ and summing the $90 \%$ threshold spectra of $B_{r}, B_{\theta}$, and $B_{z}$ from the threshold method. The curves are averaged over the total number of time steps used in their construction so they can be directly compared with one another. The first two ways match exactly, showing that the total power is being properly portioned. The $90 \%$ threshold calculation does not match exactly, though it is close. Figure 15(b) shows a comparison of the variance anistropy of the frequency power spectra as computed by the threshold and the projection methods. Again, the 


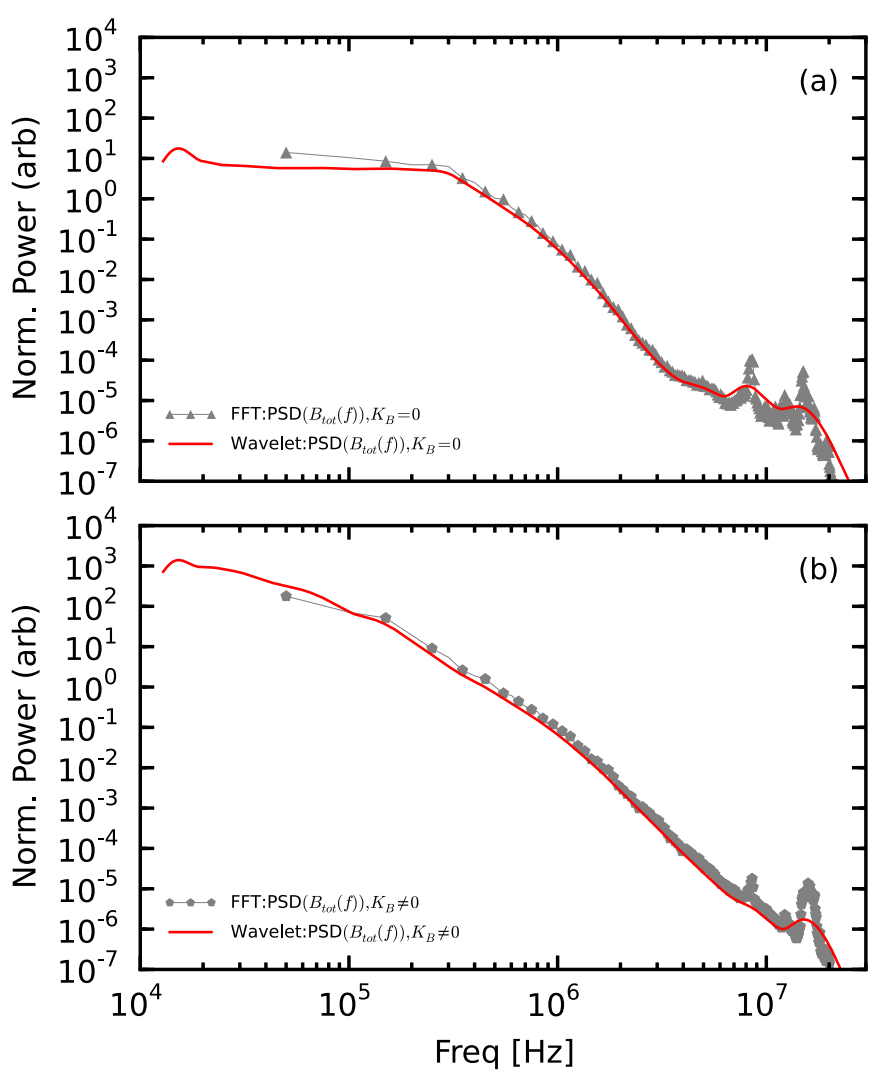

Figure 16. Comparison of magnetic power spectra constructed using a wavelet transform (red) and an FFT (gray, triangles) for zero helicity (a) and non-zero helicity (b) states.

(A color version of this figure is available in the online journal.)

curves are normalized to the total number of time steps used in construction. The quantitative comparison shows that the projection method works well to compute the level of anisotropy as it compares well to the more direct, robust threshold method.

\section{APPENDIX B}

\section{WAVELET VERSUS FFT}

Figure 16 demonstrates the correspondence between magnetic fluctuation spectra using the wavelet analysis discussed in Section 3 and a traditional FFT. The red curves in both Figure 16(a) and (b) are the wavelet-generated spectra using the full $120 \mu$ s of each shot for the zero and non-zero helicity states, respectively. The gray curves show a Fouriertransform-generated spectra for only the 40-60 $\mu$ s range of each shot. Clearly, the overall shape between the two sets of curves is nearly identical. Deviations occur at low frequencies where the wavelet transform can sample slightly lower frequencies since the analysis uses the entire time range rather than a time subset. The nature of the wavelet transform also allows for higher resolution binning especially at these lower frequencies. The wavelet transform, especially with the particular mother wavelet used-Morlet - tends to cause some smoothing in frequency space compared with the FFT. This is clearly seen at higher frequencies as modes around $10 \mathrm{MHz}$ are more clearly observed in the FFT curve than in the wavelet. However, for this particular data set, these modes are not pertinent as they are caused by characteristics of the gun system and not by turbulence physics.

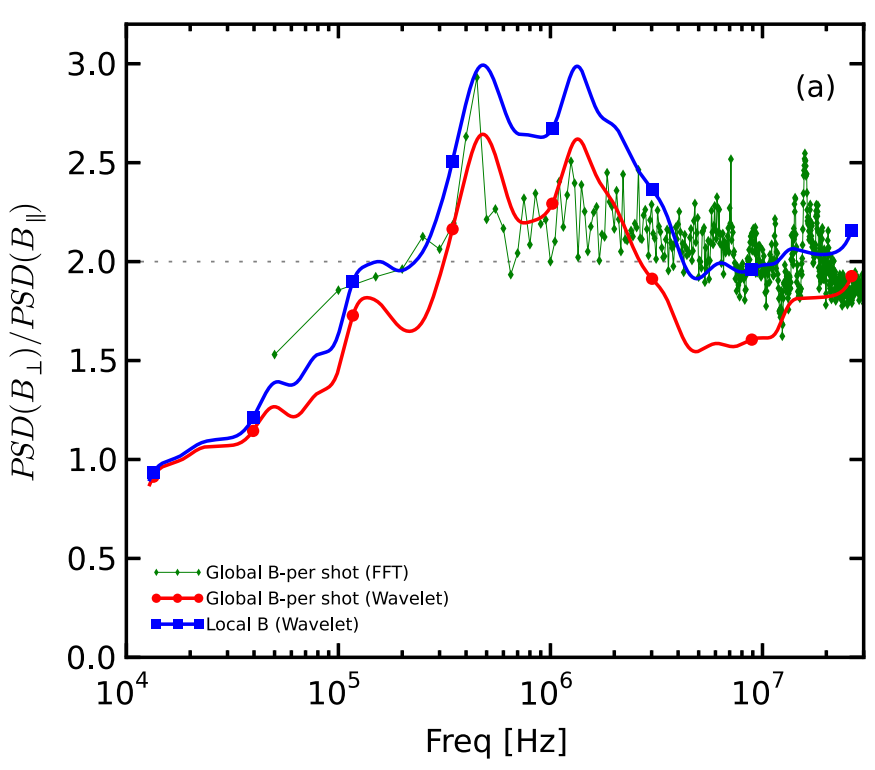

Figure 17. Comparison of the anisotropy ratio for magnetic power spectra constructed when using either a local (blue, squares) or global (red, circles) definition of the mean field. The thin green curve shows the anisotropy with a global mean field using an FFT transform rather than a wavelet.

(A color version of this figure is available in the online journal.)

\section{APPENDIX C}

\section{LOCAL VERSUS GLOBAL MAGNETIC FIELD}

The use of a temporally local versus global magnetic field in anisotropy analysis in the solar wind has been often debated (Podesta 2009; Matthaeus et al. 2012). In this paper, a local magnetic field reference vector has been used, but a relative global field can also be used to establish anisotropy. Since the experimental data is extracted on a shot-by-shot basis, the global field in this case is the mean field for the time duration of each shot. Figure 17 shows the anisotropy ratio for local field (reference vector at each time step) and global field (mean field for each shot). While the local field yields a ratio that is slightly higher than for the global, the trend as a function of frequency is clearly similar. This comparison can be extended to the FFT analysis which typically would not be useful for a variance analysis technique because it does not have the time resolution that the wavelet transform does. However, since the magnetic field does not change very quickly in these plasmas, a mean field can be chosen for each shot and then used to project the full FFT spectra generated for each shot. The green curve shows this ratio, which, while not as distinct as the wavelet-generated ratio, nevertheless shows a similar trend, especially in the $\mathrm{kHz}$ to $\mathrm{MHz}$ range. These results all suggest that though the use of a global versus local field may modify the exact numerical relationship, an anisotropy trend can be observed in either case if it is present in the plasma.

\section{REFERENCES}

Alexandrova, O., Saur, J., Lacombe, C., et al. 2009, PhRvL, 103, 165003 Barnes, C. W., Fernandez, J. C., Henins, I., et al. 1986, PhFl, 29, 3415

Belcher, J. W., \& Leverett Davis, Jr. 1971, JGR, 76, 3534

Boldyrev, S. 2006, PhRvL, 96, 115002

Bondeson, A., Markon, G., An, Z. G., et al. 1981, PhFl, 24, 1682

Chen, C. H. K., Boldyrev, S., Xia, Q., \& Perez, C. J. 2013, PhRvL, 110, 225002

Chen, C. H. K., Salem, C. S., Bonnell, J. W., Mozer, F. S., \& Bale, D. S. 2012, PhRvL, 109, 035001

Clauset, A., Rohilla Shalizi, C., \& Newman, M. E. J. 2009, SIAMR, 51, 661 
Coles, W. A., \& Harmon, K. J. 1989, ApJ, 337, 1023

Dasso, S., Milano, L. J., Matthaeus, W. H., \& Smith, W. C. 2005, ApJL, 635, L181

Dudok de Wit, T., Alexandrova, O., Furno, I., Sorriso-Valvo, L., \& Zimbardo, G. 2013, SSRv, 178, 665

Goldstein, M. L., Roberts, D. A., \& Matthaeus, H. W. 1995, ARA\&A, 33, 283

Goldreich, P., \& Sridhar, S. 1995, ApJ, 438, 763

Hamilton, K., Smith, C. W., Vasquez, B. J., \& Leamon, J. R. 2008, JGR, 113, A01106

Harmon, J. K., \& Coles, A. W. 2005, JGR, 110, A03101

He, J.-S., Marsch, E., Tu, C.-Y., et al. 2011, JGR, 116, A06207

Horbury, T. S., Forman, M., \& Oughton, S. 2008, PhRvL, 101, 175005

Horbury, T. S., Wicks, R. T., \& Chen, C.H.K. 2012, SSRv, 172, 325

Howes, G. G., Drake, D. J., Nielson, K. D., et al. 2012, PhRvL, 109, 255001

Jarboe, R. T. 1994, PPCF, 36, 945

Kiyani, K. H., Chapman, S. C., Sahraoui, F., et al. 2013, ApJ, 763, 10

Klein, K. G., Howes, G. G., \& TenBarge, J. M. 2014, ApJL, in press

Klein, K. G., Howes, G. G., TenBarge, J. M., et al. 2012, ApJ, 755, 159

Leake, J. E., Lukin, V. S., Linton, M. G., \& Meier, E. T. 2012, ApJ, 760, 109

Liewer, C. P. 1985, NucFu, 25, 543

Lukin, V. S., \& Linton, M. G. 2011, NPGeo, 18, 871

Matthaeus, W. H., Goldstein, M. L., \& Roberts, A. D. 1990, JGR, 95, 20673

Matthaeus, W. H., Servidio, S., Dmitruk, P., et al. 2012, ApJ, 750, 103

Montgomery, D., \& Turner, L. 1981, PhFl, 24, 825

Perri, S., Yordanova, E., Carbone, V., et al. 2009, JGR, 114, A02102

Podesta, J. J. 2009, ApJ, 698, 986

Podesta, J. J., Roberts, D. A., \& Goldstein, L. M. 2007, ApJ, 664, 543
Ren, Y., Almagri, A. F., Fiksel, G., et al. 2011, PhRvL, 107, 195002

Roberts, A. D. 2010, JGR, 115, A12101

Robinson, D. C., \& Rusbridge, G. M. 1971, PhFl, 14, 2499

Saharoui, F., Belmont, G., Rezeau, L., et al. 2006, PhRvL, 96, 075002

Sahraoui, F., Goldstein, M. L., Belmont, G., Canu, P., \& Rezeau, L. 2010 PhRvL, 105, 131101

Sahraoui, F., Goldstein, M. L., Robert, P., \& Khotyaintsev, Yu. V. 2009, PhRvL, 102,231102

Sarkar, A., Bhattacharjee, A., \& Ebrahimi, F. 2014, ApJ, 783, 65

Schaffner, D. A., Lukin, V. S., \& Brown, M. R. 2014a, PPCF, 56, 064003

Schaffner, D. A., Wan, A., \& Brown, M. R. 2014b, PhRvL, 112, 165001

Smith, C. W., Vasquez, B. J., \& Hamilton, K. 2006, JGR, 111, A09111

Sorriso-Valvo, L., Carbone, V., Veltri, P., et al. 1999, GeoRL, 26, 18011804

Taylor, J. B. 1986, RvMP., 58, 741

TenBarge, J. M., Podesta, J. J., Klein, K. G., \& Howes, G. G. 2012, ApJ, 753,107

Torrence, C., \& Compo, G. P. 1998, BAMS, 79, 6178

Tu, C.-Y., \& Marsch, E. 1995, SSRv, 73, 1

Tynan, G. R., Fujisawa, A., \& McKee, G. 2009, PPCF, 51, 113001

Wicks, R. T., Horbury, T. S., Chen, C. H. K., \& Schekochihin, A. A. 2010, MNRAS, 407, L31

Yordanova, E., Vaivads, A., Andre, M., Buchert, S. C., \& Voros, Z. 2008, PhRvL, 100, 205003

Zhang, X., Dandurand, D., Gray, T., Brown, M. R., \& Lukin, V. S. 2011, RScI, 82,033510

Zhou, Y., Matthaeus, W. H., \& Dmitruk, P. 2004, RvMP, 76, 1015 